Bundesgesundheitsbl 2015 · 58:1175-1191

DOI 10.1007/s00103-015-2217-3

Online publiziert: 1. September 2015

c) Springer-Verlag Berlin Heidelberg 2015

DrossMark

Bekanntmachung des Umweltbundesamtes

\title{
Stoffmonographie für
}

N-Methyl-2-pyrrolidon (NMP) und

„Human-Biomonitoring"-Werte für

die Metaboliten 5-Hydroxy-NMP und

2-Hydroxy-N-methylsuccinimid im

Urin von Erwachsenen und Kindern

\section{Stellungnahme der Kommission „Human- Biomonitoring" des Umweltbundesamtes}

\section{Einleitung}

1-Methyl-2-pyrrolidon (auch N-Methyl2-pyrrolidon, NMP; Strukturformel siehe - Abb. 1) wird als polares aprotisches Lösemittel für viele technische Anwendungen verwendet, unter anderem als Lösemittel in der Petrochemie, als reaktives Medium bei chemischen Synthesen und Lösevermittler in der pharmazeutischen Industrie sowie zu Reinigungszwecken in der Mikroelektronik. Eine Exposition am Arbeitsplatz oder auch der Allgemeinbevölkerung kann sich aus der Verwendung als Lösemittel zum Entfernen von Farben und Graffiti ergeben, in Innenräumen aus dem Einsatz in Farben und Tinten sowie in Auslegeware und Teppichen. Auch kann eine Exposition aus der Verwendung von NMP in Kosmetika und Pharmazeutika resultieren [1-3].

Für NMP (CAS-Nr. 872-50-4, EC-Nr. 212-828-1) liegen umfangreiche Daten zur Toxikologie vor. Zusammenfassende Darstellungen relevanter Untersuchungen und Bewertungen finden sich in zahlreichen Berichten und Übersichtsarbeiten [3-13] sowie im Registrierungsdossier zu NMP [14]. Aktuelle Zusammenstellungen bieten insbesondere zwei Berichte, die im Rahmen der derzeit diskutierten Einstufung und Kennzeichnung sowie Regulie- rung im Bereich der Europäischen Union vorgelegt wurden $[15,16]$.

\section{Physikochemische Eigenschaften}

NMP ist eine farblose bis schwach gelbliche, basisch reagierende Flüssigkeit mit schwach aminartigem, an Fisch erinnernden Geruch. Die Substanz ist hygroskopisch, vollständig mit Wasser mischbar und in den meisten anderen Lösemitteln löslich [13]. NMP ist beständig gegen Wasser und wird an der Luft nur langsam oxidiert [2,3].

Die wichtigsten physikochemischen Daten für NMP sind in $\bullet$ Tab. 1 zusammengestellt.

\section{Exposition der Allgemeinbevölkerung}

Erste Hinweise auf eine Hintergrundbelastung der Allgemeinbevölkerung durch NMP berichteten Koslitz et al. [17] sowie Weiß et al. [18]. Im Urin von 9 nicht be-

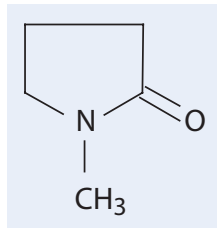

Abb. 1 Strukturformel von N-Methyl-2-pyrrolidon (NMP) 


\begin{tabular}{|c|c|}
\hline Eigenschaft & Wert \\
\hline Zustand & Flüssig, farblos bis schwach gelblich \\
\hline Schmelzpunkt & $-23,5^{\circ} \mathrm{C}$ \\
\hline Siedepunkt & $204,3^{\circ} \mathrm{C}(1013 \mathrm{hPA})$ \\
\hline Dichte & $1,028 \mathrm{~g} / \mathrm{cm}^{3}\left(20^{\circ} \mathrm{C}\right)$ \\
\hline Dampfdruck & $32 \mathrm{~Pa}\left(20^{\circ} \mathrm{C}\right), 45 \mathrm{~Pa}\left(25^{\circ} \mathrm{C}\right)$ \\
\hline Verteilungskoeffizient $\log \mathrm{K}_{\mathrm{ow}}$ & $-0,46$ \\
\hline Wasserlöslichkeit & Vollständig mischbar \\
\hline $\mathrm{pK}_{\mathrm{s}}$ & $0,93\left(25^{\circ} \mathrm{C}\right)$ \\
\hline
\end{tabular}

ruflich belasteten Kontrollpersonen wurden für den NMP-Metaboliten 5-Hydroxy-N-methylpyrrolidon (5-HNMP) Konzentrationen im Bereich von unterhalb der Nachweisgrenze $(23,0 \mu \mathrm{g} / \mathrm{L})$ bis $\mathrm{zu}$ $69 \mu \mathrm{g} / \mathrm{L}$ und für den Metaboliten 2-Hydroxy-N-methylsuccinimid (2-HMSI) von ebenfalls unterhalb der Nachweisgrenze $(15,0 \mu \mathrm{g} / \mathrm{L})$ bis $116 \mu \mathrm{g} / \mathrm{L}$ bestimmt.

Eine weitere Untersuchung durch dieselbe Arbeitsgruppe [19] wurde an insgesamt 56 Personen aus der Allgemeinbevölkerung ohne bekannte berufliche NMP-Exposition durchgeführt. 5-HNMP konnte in 96,4\% der Proben nachgewiesen werden (Nachweisgrenze, LOD: $20,0 \mu \mathrm{g} / \mathrm{L}$ ), 2-HMSI in 98,2\% (LOD: $5,0 \mu \mathrm{g} / \mathrm{L})$. Dabei lagen die Konzentrationen im Median bei 69,5 bzw. 63,5 $\mu \mathrm{g} / \mathrm{L}$, die Maximalwerte bei 620,0 bzw. 256,2 $\mu \mathrm{g} / \mathrm{L}$.

\section{Allgemeines toxikologisches Profil}

NMP bildet an der Luft typischerweise Aerosole; dabei nimmt die Tendenz zur Aerosolbildung mit steigender NMPKonzentration und Luftfeuchtigkeit sowie abnehmender Temperatur zu. In absolut trockener Luft liegt die Dampfsättigung bei Raumtemperatur bei etwa 1300 mg/ $\mathrm{m}^{3}$, bei Raumtemperatur und $60 \%$ rel. Feuchte ist ab ca. $410 \mathrm{mg} / \mathrm{m}^{3}$ (100 ppm) mit Aerosolbildung zu rechnen, bei $100 \%$ rel. Feuchte liegt ausschließlich Aerosol vor $[5,20]$. Konzentrationen über $400 \mathrm{mg}$ $\mathrm{NMP} / \mathrm{m}^{3}$ wurden in vielen Tierversuchen eingesetzt, in denen jedoch zugleich nähere Angaben darüber fehlen, ob bzw. in welchem Umfang es zur Bildung von Aerosolen in der Expositionsatmosphäre kam. Damit muss mit der Deposition von NMP-Aerosolen auf der Haut bzw. dem Fell der Versuchstiere gerechnet wer- den. Bei der hohen Resorption von NMP durch die Haut führt dies bei Ganzkörperexposition zu einer zusätzlichen, aber in den Versuchen nicht quantifizierten Aufnahme in den Körper. In einem Teil der Inhalationsstudien mit NMP wurde dieser Schwierigkeit dadurch begegnet, dass die Tiere ausschließlich im Kopfbereich („head only“ or „nose only“) exponiert wurden.

\subsection{Toxikokinetik}

\subsubsection{Tierexperimentelle Befunde}

NMP wird über die Atemwege, oral und dermal rasch und praktisch vollständig aufgenommen [7, 12]. Befunde an Ratten zur Toxikokinetik ergaben nach oraler, dermaler bzw. intravenöser Verabreichung vergleichbare Ergebnisse. Demnach wird NMP rasch resorbiert, metabolisiert und binnen 24 Stunden zu 80-90\% in Form von Metaboliten im Urin ausgeschieden [21].

Aufgenommenes NMP verteilt sich in alle Organe. In einer Untersuchung an männlichen Ratten wurden die höchsten Konzentrationen in Leber, Galle und Dünndarm sowie Nieren, Magen und Hoden gefunden [21]. In einer weiteren Studie an Ratten traten die höchsten Gehalte 4 Stunden nach der intraperitonealen Verabreichung in Muskel- und Fettgewebe sowie Leber und den Hoden auf, geschlechtsbedingte Unterschiede in der Verteilung bestanden nicht [22]. In einer Untersuchung mit inhalativer Exposition von Ratten wurde gezeigt, dass NMP die Plazentaschranke überwindet und im fetalen Blut eine ebenso hohe Konzentration erreicht wie im mütterlichen Blut [12].

Die Metabolisierung erfolgt sehr rasch in der Leber unter sukzessiver Oxidation des Pyrrolidonrings. Dabei wird im ersten Schritt 5-Hydroxy-N-methylpyrrolidon (5-HNMP) gebildet, das über N Methylsuccinimid (NMSI) weiter zu 2-Hydroxy-N-methylsuccinimid (2-HMSI) oxidiert wird (• Abb. 2). Unabhängig vom Aufnahmepfad stellt sowohl bei der Ratte wie beim Menschen (s. u.) 5-HNMP den Hauptmetaboliten dar, gefolgt von 2-HMSI [15, 23]. Bei beiden Spezies konnte außerdem in geringer Konzentration 2-Pyrrolidon im Blut und Urin nachgewiesen werden, das durch Demethylierung von NMP gebildet worden sein könnte [24].

Die Ausscheidung erfolgt weitestgehend über die Nieren mit dem Urin in Form der genannten Metaboliten (80\%) und nur zu einem geringen Teil (ca. 5\%) mit den Faeces [22]. Dabei entfallen nach Untersuchungen an Ratten unabhängig vom Aufnahmepfad knapp $70 \%$ des relativen Anteils im Urin auf 5-HNMP, $30 \%$ auf 2 -HMSI und jeweils etwa $1 \%$ auf NMSI und unverändertes NMP [8].

\subsubsection{Befunde am Menschen}

Für den Menschen wurde aus der Differenz zwischen der NMP-Konzentration in der eingeatmeten und der ausgeatmeten Luft eine inhalative Resorption von ca. $90 \%$ ermittelt $[5,25]$.

Nach oraler Verabreichung von $100 \mathrm{mg}$ NMP an drei männliche Probanden wurden $65 \%$ der zugeführten Dosis binnen neun Tagen mit dem Urin in Form identifizierter Metaboliten und als unverändertes NMP wieder ausgeschieden. Die Identifizierung erfolgte mittels Gaschromatographie/Massenspektrometrie. $67 \%$ der ausgeschiedenen Menge entfielen auf den Metaboliten 5-HNMP, 31\% auf 2-HMSI und nur 1,2\% auf unverändertes NMP, außerdem wurden Spuren von NMSI nachgewiesen; eine Konjugation der Metaboliten mit Glukuronsäure oder Sulfat erfolgte nicht. Die Halbwertszeiten der Elimination für 5-HNMP, NMSI und 2-HMSI lagen bei etwa 4, 8 bzw. 17 Stunden [23].

In einer Untersuchung an 16 männlichen Probanden, die 8 Stunden lang ( $2 \times 4$ h mit $30 \mathrm{~min}$ Pause) $80 \mathrm{mg}$ NMP $/ \mathrm{m}^{3}$ in Gasform entweder als Ganzkörperexposition oder ausschließlich über die Haut ausgesetzt waren, wurde die Aufnahme 
von NMP anhand der Ausscheidung der beiden Hauptmetaboliten 5-HNMP und 2-HMSI erfasst [26]. Bei gleichzeitiger inhalativer und perkutaner Exposition trat im Vergleich zur alleinigen inhalativen Exposition der Spitzenwert der Metaboliten im Urin später auf, und die Halbwertszeit der Elimination wurde verlängert. Von der Menge der Metaboliten, die bei Ganzkörperexposition binnen 48 Stunden nach Expositionsbeginn insgesamt im Urin ausgeschieden wurde, entfielen in Ruhe $42 \%$, unter moderater körperlicher Belastung immer noch 33\% auf den dermal resorbierten Anteil.

Aufgrund des geringen Dampfdrucks und der hohen Wasserlöslichkeit von NMP ist bei hohen Konzentrationen und hoher Luftfeuchtigkeit die Bildung von Aerosolen nicht auszuschließen, die als Kondensat auf die Haut gelangen und dort resorbiert werden können [7, 20]. In einer Probandenstudie an 6 männlichen Freiwilligen, die 8 Stunden $20 \mathrm{mg} \mathrm{NMP} / \mathrm{m}^{3}$ in feuchter ( $80 \%$ rel. Feuchte) oder trockener Luft (20\%) ausgesetzt worden waren, zeigten sich interindividuelle Unterschiede der inneren Belastung, die anhand von Plasmaspiegeln und der Ausscheidung im Urin erfasst wurde. Jedoch bestanden keine signifikanten Unterschiede zwischen beiden Expositionsbedingungen hinsichtlich Spitzenkonzentrationen in Plasma und Urin, Gesamtexkretion oder AUC [24]. Diese Befunde sprechen dafür, dass bei der eingesetzten Konzentration noch keine Aerosolbildung auftrat.

Nach Untersuchungen am Menschen wird bei 8-stündiger inhalativer Exposition der Maximalwert an NMP im Blut mit Expositionsende erreicht, der des 5-HNMP 2 Stunden später, der des NMSI 4 Stunden und der des 2-HMSI 16 Stunden später. Die Halbwertszeiten im Plasma liegen für die vier genannten Stoffe bei 4, 6, 8 und 16 Stunden $[13,25,27,28]$.

Die Ausscheidung erfolgt weitestgehend über die Nieren. In einer Studie an 16 männlichen Probanden, die $2 \times 4$ Stunden (mit 30 min Unterbrechung) gegenüber $10 \mathrm{mg} / \mathrm{m}^{3}$ exponiert worden waren, entfielen auf den binnen 48 Stunden im Urin ausgeschiedenen Anteil im Mittel $66 \%$ auf 5 -HNMP, $31 \%$ auf 2 -HMSI und knapp $1 \%$ auf NMP. Mit steigender Konzentration (bis $80 \mathrm{mg} / \mathrm{m}^{3}$ ) nahm der An-

Bundesgesundheitsbl 2015 · 58:1175-1191 DOI 10.1007/s00103-015-2217-3

c) Springer-Verlag Berlin Heidelberg 2015

Bekanntmachung des Umweltbundesamtes

Stoffmonographie für N-Methyl-2-pyrrolidon

(NMP) und "Human-Biomonitoring"

-Werte für die Metaboliten 5-Hydroxy-NMP und

2-Hydroxy-N-methylsuccinimid im Urin von Erwachsenen

und Kindern. Stellungnahme der Kommission „Human-Biomonitoring" des Umweltbundesamtes

\section{Zusammenfassung}

1-Methyl-2-pyrrolidon (NMP) wird als Lösemittel für viele technische Anwendungen verwendet. Eine Exposition der Allgemeinbevölkerung kann sich aus der Verwendung als Inhaltsstoff in Farb- und Graffiti-Entfernern ergeben, in Innenräumen auch aus dem Einsatz in Farben sowie in Auslegeware. Wegen fruchtschädigender Wirkungen wird der Einsatz von NMP in Verbraucherprodukten in der EU reglementiert. Für die Ableitung von HBM-Werten sieht die HBM-Kommission die im Tierversuch beobachteten entwicklungstoxischen Effekte bei gleichzeitig schwacher maternal toxischer Wirkung als relevant an. Ausgehend von diesen Befunden wurden für die Metaboliten 5-Hydroxy-NMP und 2-Hydroxy-N-methylsuccinimid HBM-I-Werte von $10 \mathrm{mg} / \mathrm{l}$ Urin für Kinder und $15 \mathrm{mg} / \mathrm{l}$ für Erwachsene festgelegt. Als HBM-II-Werte wurden 30 mg/l Urin für Kinder und 50 mg/l für Erwachsene bestimmt. Bei der Bewertung von Belastungen ist wegen der ähnlichen Wirkung des strukturanalogen 1-Ethyl-2-pyrrolidon (NEP) die mögliche Mischexposition gegenüber beiden Stoffen zu berücksichtigen.

Schlüsselwörter

1-Methyl-2-pyrrolidon · NMP · HBM-Werte . Human-Biomonitoring $\cdot$ HBM-Kommission

\section{Monograph for N-Methyl-pyrrolidone (NMP) and human biomonitoring values for the metabolites 5-Hydroxy-NMP and 2-Hydroxy-N-methylsuccinimide}

\section{Abstract}

1-Methyl-pyrrolidone (NMP) is used as a solvent in many technical applications. The general population may be exposed to NMP from the use as ingredient in paint and graffiti remover, indoors also from use in paints and carpeting. Because of developmental toxic effects, the use of NMP in consumer products in the EU is regulated. The developmental effects accompanied by weak maternally toxic effects in animal experiments are considered as the critical effects by the German HBM Commission. Based on these effects, $\mathrm{HBM}-\mathrm{I}$ values of $10 \mathrm{mg} / \mathrm{l}$ urine for children and of $15 \mathrm{mg} / \mathrm{l}$ for adults, respectively, were derived for the metabolites 5-Hydroxy-NMP and 2-Hydroxy-N-methylsuccinimide. HBMII-values were set to $30 \mathrm{mg} / \mathrm{l}$ urine for children and $50 \mathrm{mg} / \mathrm{l}$ for adults, respectively. Because of similar effects of the structural analogue 1-ethyl-2-pyrrolidone (NEP), the possible mixed exposure to both compounds has to be taken into account when evaluating the total burden.

\section{Keywords \\ 1-Methyl-2-pyrrolidone - NMP · HBM values . Human Biomonitoring $\cdot$ HBM Commission}

teil unveränderten NMPs geringfügig zu $(1,5 \%)$, der des 5-HNMP leicht ab (61\%) [29]. Die Halbwertszeit der Elimination mit dem Urin liegt nach Inhalation mit Ganzkörperexposition in Ruhe und bei leichter körperlicher Aktivität (75 W) für NMP bei etwa 4 Stunden, für 5-HNMP bei 6,5 Stunden und für 2-HMSI bei 22 Stunden. Bei alleiniger dermaler Ganzkörperexposition verlängert sich die Halbwertszeit für 5-HNMP um etwa 20\%, vermut- lich aufgrund der fortgesetzten systemischen Verfügbarkeit aus der Haut nach Ende der Exposition [26].

In ihrer neuesten Bewertung [9] hat die Senatskommission zur Prüfung gesundheitsschädlicher Arbeitsstoffe die Möglichkeiten zur Ableitung tolerabler Konzentrationen von NMP am Arbeitsplatz auf Basis eines PBPK-Modells und einer Benchmarkdosis-Berechnung [30] diskutiert, dieses Modell jedoch wegen 


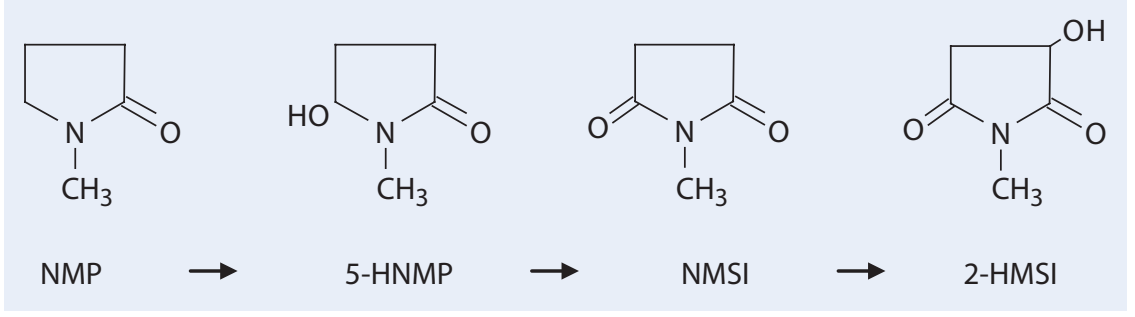

Abb. $2 \Delta$ Metabolismus von NMP bei Ratten und Menschen

verschiedener Mängel und Unklarheiten wieder verworfen.

Die Ausscheidung der Metaboliten mit dem Urin wird herangezogen, um die innere Belastung mit NMP am Arbeitsplatz $\mathrm{zu}$ erfassen. Zur Beurteilung wurden ein BAT-Wert für die Ausscheidung von 5-HNMP [8] sowie ein BLV für 5-HNMP und 2-HMSI im Urin abgeleitet und festgelegt [12].

\subsection{Akute Toxizität}

Es liegen Daten aus dem Registrierungsdossier [14] sowie aus dem OECD-SIDS [10] vor, die vom RIVM im Annex-XVBericht zusammengefasst wurden [15]. Die folgenden Angaben wurden diesem RIVM-Bericht entnommen.

Die akute Toxizität von NMP ist bei allen untersuchten Verabreichungswegen gering. Nach oraler Verabreichung wurden bei Mäusen und Ratten $\mathrm{LD}_{50}$-Werte zwischen 3605 und 7725 mg/kg KG ermittelt, nach dermaler Applikation bei Ratten lagen die $\mathrm{LD}_{50}$-Werte mit 5000-7000 mg/ $\mathrm{kg}$ KG in einem ähnlichen Bereich. In Inhalationsstudien zur Prüfung auf akute Toxizität wurde ein Aerosol-Dampf-Gemisch eingesetzt. In einer Untersuchung mit $87 \%$ einatembaren Aerosolanteil lag die $\mathrm{LC}_{50}$ bei $>5100 \mathrm{mg} / \mathrm{m}^{3}$.

\subsection{Haut- und Schleimhautreizung}

Es liegen Daten aus dem Registrierungsdossier [14] sowie aus dem OECD-SIDS [10] tierexperimentelle Befunde und $\mathrm{Hu}$ mandaten vor, die vom RIVM im AnnexXV-Bericht zusammengefasst wurden [15]. Die folgenden Angaben wurden diesem RIVM-Bericht entnommen.

Bei Kaninchen führten $0,5 \mathrm{ml}$ unter okklusiven Bedingungen auf der intakten und der aufgescheuerten Haut nur zu leichter vorübergehender Rötung. Auch in einem modifizierten Draize-Test an Kaninchen mit $20 \%$ NMP in öliger Lösung war nur eine vorübergehende minimale Reizung zu verzeichnen. Im Auge von Kaninchen verursachte $0,1 \mathrm{ml}$ flüssiges NMP hingegen ausgeprägte, aber reversible Reizeffekte in Form von Bindehautrötung, Hornhauttrübung und Iritis.

In einer subchronischen Inhalationsstudie über 3 Monate führten Konzentrationen ab 1000 mg/m³ bei Ratten zu Reizungserscheinungen der Atemwege, die NOAEC lag bei $500 \mathrm{mg} / \mathrm{m}^{3}$.

Beim Menschen lieferte ein Patch-Test an 50 Probanden keine Hinweise auf hautreizende Wirkung während der 24stündigen Einwirkung von NMP. Dagegen wird berichtet, dass Arbeiter nach mehrtägigem Kontakt mit flüssigem NMP, das sie mit Papiertüchern von Plastikteilen abgewischt hatten, Hautreizungen zeigten. Nach einem weiteren Bericht traten nach mehrmaligem Hautkontakt nach drei Tagen Schwellungen und Runzelbildung der Haut auf, jedoch keine Reizeffekte.

Die inhalative Exposition gegenüber bis zu $50 \mathrm{mg} \mathrm{NMP} / \mathrm{m}^{3}(2 \times 4 \mathrm{~h}$ mit $30 \mathrm{~min}$ Pause) verursachte bei den 6 exponierten Probanden weder subjektive Reizwirkungen an Augen, Nase und Atemwegen noch Veränderungen von Lungenfunktion und Nasenhöhle (Rhinometrie) [27]. In einer weiteren Probandenstudie an 16 Personen verursachten bis zu $80 \mathrm{mg} \mathrm{NMP/}$ $\mathrm{m}^{3}$ ( $2 \times 4$ h mit 30 min Pause) oder auch $25 \mathrm{mg} / \mathrm{m}^{3}$ mit viermal $15 \mathrm{~min}$ Spitzen von je $160 \mathrm{mg} / \mathrm{m}^{3}$ keine Augenreizung [31]. In einer weiteren Studie mit identischem Protokoll berichteten die Probanden über Geruchsbelästigung, trigeminal vermittelte Reizungen traten jedoch nicht auf [32, 33].

\subsection{Sensibilisierung}

In zwei nicht gemäß OECD-Richtlinien ausgeführten Untersuchungen an Meerschweinchen wurde keine hautsensibilisierende Wirkung festgestellt. In einem Patch-Test an 50 Probanden traten nach insgesamt 15 ganztägigen Kontakten leichte bis mäßige, vorübergehende Hautreizungen auf, jedoch keine Anzeichen einer Kontaktsensibilisierung [15].

\subsection{Toxizität bei wiederholter Verabreichung}

Die Befunde von kombinierten chronischen Toxizitäts-/Kanzerogenitätsstudien sind im Kapitel 4.7 beschrieben.

\section{Untersuchungen mit oraler Exposition}

Es liegen Befunde an Ratten, Mäusen und Hunden vor (• Tab. 2).

In einer subakuten Studie erhielten je 5 männliche und weibliche Sprague-Dawley-Ratten/Dosis $28 \mathrm{~d}$ lang 0, 2000, 6000, 18000 oder 30000 ppm NMP im Futter (Männchen/Weibchen ca. 0, 149/161, 429/493, 1234/1548, 2019/2269 mg/(kg $\mathrm{KG} \times \mathrm{d})[13,34]$. Bei den Männchen traten ab 18000 ppm (1234 mg/(kg KG × d)), bei den Weibchen ab 30000 ppm $(2269 \mathrm{mg} /(\mathrm{kg} \mathrm{KG} \times \mathrm{d}))$ substanzbedingte Wirkungen auf (Körpergewicht und Futteraufnahme vermindert, Veränderungen klinisch-chemischer Parameter, insbesondere verminderter Albumin- und Glucosegehalt im Blut). Weiterhin zeigte sich eine leichte Lymphopenie, verminderte Zelldichte im Knochenmark und Thymusatrophie, die von den Autoren ebenfalls als behandlungsbedingt angesehen werden, aber auch ein Sekundäreffekt der verminderten Nahrungsaufnahme sein könnten. In dieser Studie lag die NOAEC bei 6000 ppm NMP im Futter (NOAEL: $429 \mathrm{mg} /(\mathrm{kg} \mathrm{KG} \times \mathrm{d})$, LOAEL: $1234 \mathrm{mg} /(\mathrm{kg} \mathrm{KG} \times \mathrm{d}))$.

In einer Studie der BASF [35] erhielten je 10 männliche und weibliche Sprague-Dawley-Ratten 28 Tage lang mit der Schlundsonde 0, 259, 517, 1033 bzw. 2066 mg NMP/(kg KG×d). Bei den Männchen war ab $517 \mathrm{mg} \mathrm{NMP/(kg} \mathrm{KG} \times$ d) die Gewichtszunahme vermindert, bei höheren Dosierungen zeigten sich außerdem Veränderungen im Nieren- und Le- 
Tab. 2 Zusammenstellung der NOAEL und LOAEL nach subakuter bis subchronischer oraler Verabreichung von NMP

\begin{tabular}{|c|c|c|c|c|c|}
\hline $\begin{array}{l}\text { Spezies, Stamm, Anzahl und } \\
\text { Geschlecht/Dosis }\end{array}$ & Exposition & $\begin{array}{l}\text { NOAEL } \\
{[\mathrm{mg} /(\mathrm{kg} \mathrm{KG} \times \mathrm{d})]}\end{array}$ & $\begin{array}{l}\text { LOAEL } \\
{[\mathrm{mg} /(\mathrm{kg} \mathrm{KG} \times \mathrm{d})]}\end{array}$ & $\begin{array}{l}\text { Effekt, Veränderungen beim } \\
\text { LOAEL }\end{array}$ & Quelle \\
\hline Ratte, Sprague-Dawley, 5 M/5 W & Subakut, $28 d$ & 429 & 1234 & $\begin{array}{l}\text { KG vermindert, klinisch-chemi- } \\
\text { sche Parameter verändert }\end{array}$ & [34] \\
\hline Ratte, Sprague-Dawley, 10 M/10 W & Subakut, $28 \mathrm{~d}$ & 259 & 517 & Gewichtszunahme vermindert & [35] \\
\hline Ratte, Sprague-Dawley, 20-26 M/W & Subchronisch, $90 \mathrm{~d}$ & 169/217 (M/W) & 433/565 (M/W) & Gewichtszunahme vermindert & [36] \\
\hline Maus, B6C3F1, 5 M/5W & Subakut, $28 \mathrm{~d}$ & 820 & 2500 & Nierenschäden & [37] \\
\hline Maus, B6C3F1, $10 \mathrm{M} / 10 \mathrm{~W}$ & Subchronisch, $90 \mathrm{~d}$ & 277 & 619 & $\begin{array}{l}\text { Lebergewicht erhöht, klinisch- } \\
\text { chemische Parameter verändert }\end{array}$ & [36] \\
\hline Hund, Beagle, $6 \mathrm{M} / 6 \mathrm{~W}$ & Subchronisch, $90 \mathrm{~d}$ & 250 & - & NOAEL = höchste getestete Dosis & [38] \\
\hline
\end{tabular}

bergewicht, jedoch ohne histopathologischen Befund. Bei der höchsten Dosis traten Hodenschäden mit Degeneration des Keimepithels auf (NOAEL: $259 \mathrm{mg} /(\mathrm{kg}$ $\mathrm{KG} \times \mathrm{d})$, LOAEL: $517 \mathrm{mg} /(\mathrm{kg} \mathrm{KG} \times \mathrm{d}))$.

Eine kombinierte subchronische Studie/Neurotoxizitätsstudie wurde an Sprague-Dawley-Ratten durchgeführt [36]. Dazu erhielten Gruppen von 20-26 Männchen und Weibchen drei Monate 0, 3000, 7500 oder 18000 ppm NMP im Futter (Männchen/Weibchen ca. 0, 169/217, 433/565, 1057/1344 mg/(kg KG × d)). Je 10 Tiere pro Geschlecht aus der Kontrollgruppe und der mit der höchsten Dosierung wurden einen Monat lang nach Expositionsende nachbeobachtet. Dosen ab 7500 ppm führten zu verminderter $\mathrm{Ge}$ wichtszunahme und Futteraufnahme, ab 18000 ppm waren Leber- und Nierengewicht ohne histopathologisch erkennbare Veränderungen erhöht. Bei dieser Dosis traten in der Prüfung auf neurotoxische Effekte bei Männchen außerdem Veränderungen im Foot-Splay-Test (am Ende der Nachbeobachtungsphase nicht mehr nachweisbar) und sedierende Effekte auf (NOAEC: 3000 ppm NMP entsprechend NOAEL: 169/217 mg/(kg KG $\times$ d), LOAEL: 433/565 mg/(kg KG $\times$ d) $)$.

Bei B6C3F1-Mäusen (je 5 Männchen und Weibchen/Dosis) hatte die subakute Exposition über 28 Tage mit 0, 500, 2500, 7500 oder 10000 ppm NMP im Futter (ca. 0, 160, 820, 2500, 3370 mg/(kg KG $\times$ d)) keinen Einfluss auf Futteraufnahme und Gewichtsentwicklung [37]. Veränderungen zeigten sich in der Niere in Form wolkiger Schwellungen im distalen Tubulusepithel ab 7500 ppm NMP (Männchen) und bei 10000 ppm NMP (Weibchen). Bei der höchsten Dosierung starb ein Männchen an Nierenschäden, bei den
Weibchen fanden sich erniedrigte Alkalische Phosphatase (ALP)-Aktivitäten im Serum. Ab 2500 ppm NMP war als Zeichen der systemischen Verfügbarkeit von NMP der Urin gelblich verfärbt. Die NOAEC dieser Studie lag bei 2500 ppm NMP entsprechend NOAEL: $820 \mathrm{mg} /(\mathrm{kg} \mathrm{KG} \times$ d), LOAEL: $2500 \mathrm{mg} /(\mathrm{kg} \mathrm{KG} \times \mathrm{d})$.

In einer subchronischen Studie mit B6C3F1-Mäusen (je 10 Männchen und Weibchen/Dosis) erhielten die Tiere 0, 1000, 2500 oder 7500 ppm NMP im Futter (ca. 0, 277, 619, $1931 \mathrm{mg} /(\mathrm{kg} \mathrm{KG} \times \mathrm{d})$ ) für 3 Monate und in einer Satellitengruppe für 4 Wochen [36]. Ab 2500 ppm NMP war bei den Männchen das Lebergewicht erhöht, ab 7500 ppm NMP trat bei beiden Geschlechtern eine zentrilobuläre Hypertrophie der Leberzellen auf und in der Satellitengruppe waren klinisch-chemische Veränderungen (ALP-Aktivität sowie Triglycerid- und Cholesterolspiegel) nachweisbar (NOAEC: 1000 ppm NMP entspr. NOAEL: $277 \mathrm{mg} /(\mathrm{kg} \mathrm{KG} \times \mathrm{d})$, LOAEL: $619 \mathrm{mg} /(\mathrm{kg} \mathrm{KG} \times \mathrm{d}))$.

In einer Studie an Beagle-Hunden (je 6 Männchen und Weibchen/Dosis) erhielten die Tiere 90 Tage lang NMP im Futter (entspr. Körperdosen von 0, 25, 79 oder $250 \mathrm{mg} /(\mathrm{kg} \mathrm{KG} \times \mathrm{d}))$ [38]. Bis zur höchsten Dosierung traten keine bleibenden oder substanzbedingten Veränderungen auf, die das Ausmaß biologischer oder historischer Kontrollen überschritten (NOAEL: $250 \mathrm{mg} /(\mathrm{kg} \mathrm{KG} \times \mathrm{d}))$.

\section{Untersuchungen mit inhalativer Exposition (•Tab. 3)}

In mehreren von der BASF mit NMP durchgeführten Inhalationsstudien, die zusammengefasst durch das RIVM $[15,16]$ berichtet werden, wurde der Einfluss der Expositionsart („nose only“ vs. Ganzkör- per), der Luftfeuchtigkeit und des physikochemischen Status (Aerosol vs. Dampf) untersucht. Dazu wurden weibliche Sprague-Dawley- oder Wistar-Ratten 6 h/d, 5 $\mathrm{d} /$ Woche 2 oder 4 Wochen lang gegenüber 0 oder $1000 \mathrm{mg} / \mathrm{m}^{3} \mathrm{NMP}$ exponiert. Bei einer "nose only“-Exposition traten unabhängig von Aerosolanteil und Luftfeuchtigkeit lediglich lokale Effekte (leichte nasale Reizungen) und, als Zeichen der erfolgten Resorption und Metabolisierung des NMP, eine Färbung des Urins auf. Hingegen führte die Ganzkörperexposition gegenüber großen Aerosoltropfen bei hoher Luftfeuchtigkeit zu schweren systemisch toxischen Wirkungen bis hin zu hoher Mortalität. Feineres Aerosol führte zu weniger schweren Effekten und bedingte keine erhöhte Mortalität. Die Unterschiede werden auf erhöhte dermale und orale Exposition gegenüber den größeren Aerosoltropfen zurückgeführt, (Zum Einfluss von NMP-Konzentration und Luftfeuchtigkeit auf den Anteil an Aerosol siehe Beginn des Abschnitts 4).

In einer subakuten Studie über $28 \mathrm{~d}$ an je 5 männlichen und weiblichen WistarRatten traten nach der Exposition $(6 \mathrm{~h} / \mathrm{d}$, $5 \mathrm{~d} /$ Woche mit $0,10,30,100 \mathrm{mg} / \mathrm{m}^{3} \mathrm{Ae}$ rosol, nose only) keine behandlungsbedingten adversen Veränderungen auf. Bei der höchsten Konzentration war der Urin der Tiere verfärbt, was auf die systemische Verfügbarkeit der Substanz hinweist [39].

In einer weiteren subakuten Studie wurden Sprague-Dawley-Ratten (je 15 Männchen und Weibchen/Dosis) $6 \mathrm{~h} / \mathrm{d}$, $5 \mathrm{~d} /$ Woche gegenüber einem AerosolDampf-Gemisch von NMP ganzkörperexponiert $\left(0,100,500,1000 \mathrm{mg} / \mathrm{m}^{3}\right)$ [40]. Die NMP-Exposition führte bei allen Tieren nach 3-4 Stunden zu Lethargie und Atemproblemen, die bei der höchsten Do- 


\begin{tabular}{|c|c|c|c|c|c|}
\hline $\begin{array}{l}\text { Spezies, Stamm, Anzahl } \\
\text { und Geschlecht/Dosis }\end{array}$ & Exposition & $\begin{array}{l}\text { NOAEC } \\
\left(\mathrm{mg} / \mathrm{m}^{3}\right)\end{array}$ & $\begin{array}{l}\text { LOAEC } \\
\left(\mathrm{mg} / \mathrm{m}^{3}\right)\end{array}$ & Effekt, Veränderungen beim LOAEL & Quelle \\
\hline Ratte, Wistar, $5 \mathrm{M} / 5 \mathrm{~W}$ & $\begin{array}{l}\text { Subakut, } 6 \mathrm{~h} / \mathrm{d}, 5 \mathrm{~d} / \\
\text { Woche, head only }\end{array}$ & 100 & - & NOAEC = höchste getestete Konzentration & [39] \\
\hline $\begin{array}{l}\text { Ratte, Sprague-Dawley } \\
15 \mathrm{M} / 15 \mathrm{~W}\end{array}$ & $\begin{array}{l}\text { Subakut, } 6 \mathrm{~h} / \mathrm{d}, 5 \mathrm{~d} / \\
\text { Woche, Ganzkörper }\end{array}$ & $\begin{array}{l}500 \text { (systemisch) } \\
- \text { (lokal) }\end{array}$ & $\begin{array}{l}1000 \text { (systemisch } \\
100 \text { (lokal) }\end{array}$ & $\begin{array}{l}\text { massive generelle Toxizität, Hodenatrophie, } \\
\text { Letalität, lokal: Atemstörung }\end{array}$ & [40] \\
\hline Ratte, Wistar, $10 \mathrm{M} / 10 \mathrm{~W}$ & $\begin{array}{l}\text { Subchronisch, } 90 \mathrm{~d} \text {, } \\
\text { head-only }\end{array}$ & $\begin{array}{l}500 \text { (systemisch } \\
\text { und lokal) }\end{array}$ & $\begin{array}{l}1000 \text { (systemisch } \\
\text { und lokal) }\end{array}$ & $\begin{array}{l}\text { Gewichtszunahme vermindert, Hodenschä- } \\
\text { den, hämatologische Parameter verändert }\end{array}$ & [41] \\
\hline
\end{tabular}

Tab. 4 Zusammenstellung der NOAEL und LOAEL nach subakuter dermaler Verabreichung von NMP

\begin{tabular}{|llllll}
\hline $\begin{array}{l}\text { Spezies, Stamm, Anzahl und } \\
\text { Geschlecht/Dosis }\end{array}$ & Exposition & $\begin{array}{l}\text { NOAEL } \\
(\mathrm{mg} /(\mathrm{kg} \mathrm{KG} \times \mathrm{d}))\end{array}$ & $\begin{array}{l}\text { LOAEL } \\
(\mathrm{mg} /(\mathrm{kg} \mathrm{KG} \times \mathrm{d}))\end{array}$ & $\begin{array}{l}\text { Effekt, Veränderungen } \\
\text { beim LOAEL }\end{array}$ & $\begin{array}{l}\text { Quelle } \\
\text { Kaninchen, 2 M }\end{array}$ \\
\hline
\end{tabular}

sierung bis zur nächsten Exposition noch nicht abgeklungen waren. Bei den beiden niedrigeren Konzentrationen wurden keine substanzbedingten pathologischen Veränderungen festgestellt. Berichtet wird eine leichte Hodenatrophie, $\mathrm{zu}$ der jedoch keine quantitativen Angaben gemacht werden. Bei der höchsten Dosierung starben 13 der Tiere in den ersten 9 Tagen oder wurden wegen schlechten Allgemeinzustands eingeschläfert, die überlebenden Tiere wurden nicht weiter exponiert. Diese Tiere zeigten Gewichtsverlust, fokale Pneumonien, Hypoplasie des Knochenmarks, Atrophie des lymphatischen Gewebes in Milz und Thymus sowie eine erhöhte Zahl von Neutrophilen im Blut, die Effekte waren nach Sistieren der Exposition binnen 2 Wochen reversibel. Drei der überlebenden Männchen aus der am höchsten exponierten Gruppe wiesen eine schwere Hodenatrophie auf. Aus dieser Untersuchung ergibt sich für systemische Effekte eine NOAEC von $500 \mathrm{mg} /$ $\mathrm{m}^{3}$ (LOAEC: $1000 \mathrm{mg} / \mathrm{m}^{3}$ ), eine NOAEC für lokale Effekte kann angesichts der leichten Reizeffekte bei allen Konzentrationen nicht angegeben werden.

In einer subchronischen Studie an Wistar-Ratten (je 10 Männchen und Weibchen/Dosis) erfolgte eine KopfNasen-Exposition der Tiere gegenüber NMP-Aerosol mit 0, 125, 250, 750 ppm $\left(0,500,1000\right.$ bzw. $\left.3000 \mathrm{mg} / \mathrm{m}^{3}\right)$ an $6 \mathrm{~h} / \mathrm{d}$, $5 \mathrm{~d} /$ Woche für 13 Wochen [41]. Eine Satellitengruppe wurde nach Exposition gegenüber 0 bzw. $3000 \mathrm{mg} / \mathrm{m}^{3}$ im Anschluss an die genannte Exposition über einen vierwöchigen expositionsfreien Zeitraum nachbeobachtet. Alle Konzen- trationen verursachten eine dunkelgelbe Färbung des Urins. Bei Konzentrationen ab $1000 \mathrm{mg} / \mathrm{m}^{3}$ waren am Ende der Exposition nasale Reizung mit Gewebsverkrustungen und Reizwirkungen auf die Atemwege erkennbar. Ab 1000 mg/m³ war die Körpergewichtszunahme der Männchen reduziert (bei $1000 \mathrm{mg} / \mathrm{m}^{3}$ noch nicht signifikant), das absolute Hodengewicht vermindert und das Keimepithel geschädigt. Bei beiden Geschlechtern waren bei der höchsten Konzentration hämatologische Parameter beeinträchtigt, u. a. mit erhöhter Zahl an Neutrophilen und verminderter Lymphozytenzahl. In der Satellitengruppe zeigten die Männchen am Ende der Nachbeobachtung verminderte Gewichtszunahme und eine verminderte Zellularität im Hoden (NOAEC für systemische und lokale Effekte: $500 \mathrm{mg} / \mathrm{m}^{3}$, LOAEC für systemische und lokale Effekte $1000 \mathrm{mg} / \mathrm{m}^{3}$ ).

\section{Untersuchungen mit dermaler Exposition}

In einer älteren, nicht gemäß OECDRichtlinien durchgeführten Studie an männlichen Kaninchen erhielten je 2 Tiere/Gruppe vier Wochen lang NMP einmal täglich an $5 \mathrm{~d} /$ Woche in Dosen von 0,413 , 826 bzw. $1653 \mathrm{mg} /(\mathrm{kg} \mathrm{KG} \times \mathrm{d})$ auf die intakte oder aufgeschürfte Haut. Alle NMPDosierungen verursachten leichte lokale Reizungen der Haut (• Tab. 4). Klinische, hämatologische und histopathologische Untersuchungen erbrachten keine Anzeichen systemisch-toxischer Wirkungen, auch die Gewichtsentwicklung war nicht beeinflusst. Nicht ausgeschlossen werden kann jedoch, dass der Tod eines Tieres mit aufgeschürfter Haut unter der höchsten Dosierung expositionsbedingt war. Als NOAEL für systemische Wirkungen ergäbe sich dann ein Wert von $826 \mathrm{mg} /(\mathrm{kg} \mathrm{KG} \times \mathrm{d})($ LOAEL: $1653 \mathrm{mg} /$ $(\mathrm{kg} \mathrm{KG} \times \mathrm{d}))$ [42].

\subsection{Gentoxizität}

In der Gesamtbewertung lassen die Befunde nicht auf ein gentoxisches Potential von NMP schließen.

\subsubsection{In vitro}

Bei Bakterien wurde in mehreren Studien in An- und Abwesenheit von exogenem metabolischem Aktivierungssystem aus Ratten- oder Hamsterleber an unterschiedlichen Stämmen von Salmonella typhimurium beim Testen bis in den zytotoxischen Dosisbereich keine mutagene Wirkung festgestellt. Eine minimale, nicht dosis-abhängige Zunahme von Revertanten in einem Test mit den Stämmen TA102 und TA 104 in Abwesenheit von exogenem metabolischen Aktivierungssystem konnte in einem weiteren Test an diesen Stämmen nicht bestätigt werden [3].

In Zellen der Hefe Saccharomyces cerevisiae induzierte NMP im Bereich von 7,6-23 g/L dosisabhängig Aneuploidien; dabei wirkten Konzentrationen ab 18 g/l stark zytotoxisch (> 50\% verminderte Überlebensrate) [9].

An Säugerzellen verursachte NMP in L5178Y-Lymphomzellen der Maus keine mutagenen Effekte und in Primärkulturen von Rattenleberzellen keine erhöhte außerplanmäßige DNA-Synthese [9]. In einem HPRT (Hypoxanthin-Gua- 


\begin{tabular}{|c|c|c|c|c|c|}
\hline $\begin{array}{l}\text { Spezies, Stamm, Anzahl } \\
\text { und Geschlecht/Dosis }\end{array}$ & Exposition & $\begin{array}{l}\text { NOAEL/NOAEC } \\
(\mathrm{mg} /(\mathbf{k g ~ K G} \times \mathrm{d}) \text { bzw. } \\
\left.\mathrm{mg} / \mathrm{m}^{3}\right)\end{array}$ & $\begin{array}{l}\text { LOAEL/LOAEC } \\
(\mathrm{mg} /(\mathrm{kg} \mathrm{KG} \times \mathrm{d}) \mathrm{bzw} . \\
\left.\mathrm{mg} / \mathrm{m}^{3}\right)\end{array}$ & $\begin{array}{l}\text { Effekt, Veränderungen beim } \\
\text { LOAEL/LOAEC, Kanzerogenität }\end{array}$ & Quelle \\
\hline $\begin{array}{l}\text { Ratte, Sprague-Dawley, } \\
62 \mathrm{M} / 62 \mathrm{~W}\end{array}$ & $2 \mathrm{a}$, oral, Futter & $\begin{array}{l}207 \mathrm{mg} /(\mathrm{kg} \mathrm{KG} \times \mathrm{d})(M), \\
283 \mathrm{mg} /(\mathrm{kg} \mathrm{KG} \times \mathrm{d})(\mathrm{W})\end{array}$ & $\begin{array}{l}678 \mathrm{mg} /(\mathrm{kg} \mathrm{KG} \times \mathrm{d})(\mathrm{M}) \\
939 \mathrm{mg} /(\mathrm{kg} \mathrm{KG} \times \mathrm{d})(\mathrm{W})\end{array}$ & $\begin{array}{l}\text { Gewichtszunahme vermindert, Ne- } \\
\text { phropathie (M), nicht kanzerogen }\end{array}$ & [43] \\
\hline Maus, B6C3F1, $50 \mathrm{M} / 50 \mathrm{~W}$ & $\begin{array}{l}18 \text { Monate, oral, } \\
\text { Futter }\end{array}$ & $\begin{array}{l}89 \mathrm{mg} /(\mathrm{kg} \mathrm{KG} \times \mathrm{d})(\mathrm{M}), \\
221 \mathrm{mg} /(\mathrm{kg} \mathrm{KG} \times \mathrm{d})(\mathrm{W})\end{array}$ & $\begin{array}{l}173 \mathrm{mg} /(\mathrm{kg} \mathrm{KG} \times \mathrm{d})(\mathrm{M}) \\
1399 \mathrm{mg} /(\mathrm{kg} \mathrm{KG} \times \mathrm{d})(\mathrm{W})\end{array}$ & $\begin{array}{l}\text { Gewichtszunahme vermindert, M: } \\
\text { Leberadenome +-karzinome, W: } \\
\text { Leberadenome }\end{array}$ & [43] \\
\hline $\begin{array}{l}\text { Ratte, Sprague-Dawley, } \\
120 \text { M/120 W }\end{array}$ & $\begin{array}{l}2 \mathrm{a} \text {, inhalativ, } \\
6 \mathrm{~h} / \mathrm{d}, 5 \mathrm{~d} / \text { Wo- } \\
\text { che, Ganzkörper }\end{array}$ & $40 \mathrm{mg} / \mathrm{m}^{3}$ & $400 \mathrm{mg} / \mathrm{m}^{3}$ & $\begin{array}{l}\text { Gewichtszunahme vermindert }(M) \text {, } \\
\text { klinisch-chemische Parameter ver- } \\
\text { ändert ( } M \text {, nur nach } 18 \text { Monaten) }\end{array}$ & [40] \\
\hline
\end{tabular}

nin-Phosphoribosyl-Transferase)-Test an Ovarzellen des Chinesischen Hamsters trat ebenfalls in An- und Abwesenheit von exogenem metabolischen Aktivierungssystem keine mutagene Wirkung auf [14].

\subsubsection{In vivo}

In vivo führte die einmalige orale Verabreichung unterschiedlicher Dosen von NMP bei NMRI-Mäusen im Mikronukleustest beim Testen bis in den akut toxischen Bereich (bis 3800 mg/kg KG) binnen $72 \mathrm{~h}$ weder zu aneuploiden noch klastogenen Wirkungen. Auch bei männlichen und weiblichen Chinesischen Hamstern konnten nach oraler Gabe akut toxischer Dosen von bis zu 3800 mg/kg KG binnen $24 \mathrm{~h}$ keine Chromosomenaberrationen im Knochenmark festgestellt werden. In einer Studie mit inhalativer Exposition männlicher und weiblicher Chinesischer Hamster $\left(3300 \mathrm{mg} / \mathrm{m}^{3}, 6 \mathrm{~h} / \mathrm{d}\right.$, $5 \mathrm{~d}$ /Woche, 6 Wochen) wurde ein leicht vermehrtes Vorkommen von Chromosomenaberrationen im Knochenmark festgestellt, der Effekt war jedoch nicht signifikant. In einem Dominant-Letal-Test mit i.p.-Verabreichung von $391 \mathrm{mg} / \mathrm{kg} \mathrm{KG}$ einmal wöchentlich über 8 Wochen traten vermehrt Postimplantationsverluste auf [3]. Dieser Effekt dürfte auf die entwicklungstoxische Wirkung von NMP zurückzuführen sein.

\subsection{Kanzerogenität (• Tab. 5)}

\section{Untersuchungen mit oraler Exposition}

In einer Studie mit oraler Verabreichung erhielten Sprague-Dawley-Ratten (je 62 Männchen und Weibchen/Dosis) 2 Jahre lang 0, 1600, 5000 oder 15000 ppm NMP mit dem Futter (entspr. 0; 66,4; 207;
$678 \mathrm{mg} /(\mathrm{kg} \mathrm{KG} \cdot \mathrm{d})$ bei Männchen und 0; 87,8; 283; $939 \mathrm{mg} /(\mathrm{kg} \mathrm{KG} \cdot \mathrm{d})$ bei Weibchen) [43]. Am Ende dieses Zeitraums war bei der höchsten Dosierung das Körpergewicht der Tiere gegenüber den Kontrolltieren signifikant niedriger; bei den Männchen war außerdem die Überlebenszeit im Zusammenhang mit einer chronischen fortschreitenden Nephropathie reduziert. Auch bei 5000 ppm war die Überlebenszeit der Männchen im Zusammenhang mit unterschiedlichen Veränderungen wie etwa Hypophysentumoren reduziert; wegen fehlender Dosis-WirkungsBeziehungen wurden diese Beobachtungen von den Autoren als nicht substanzbedingt bewertet. Bei der höchsten Dosis zeigten sich bei den Männchen außerdem Veränderungen der mesenterialen Lymphknoten und Veränderungen der Milz mit Makrophagenansammlung, zentrilobuläre fettige Leberdegeneration, Degeneration der Nierenrinde, Degeneration und Atrophie der Hoden sowie Oligospermie der Nebenhoden. Bei den Weibchen zeigten sich lediglich Milzveränderungen. Eine substanzbedingte erhöhte Inzidenz von Tumoren wurde nicht festgestellt (NOAEL: 207/283 mg/(kg KG × d), LOAEL 678/939 mg/(kg KG × d)).

Von derselben Arbeitsgruppe [43] wurden B6C3F1-Mäuse (je 50 Männchen und Weibchen/Dosis) mit 0, 600, 1200 oder 7200 ppm NMP im Futter behandelt, entspr. 0, 89, 173, $1089 \mathrm{mg} /(\mathrm{kg} \mathrm{KG} \times \mathrm{d})$ bei Männchen und 0, 115, 221, $1399 \mathrm{mg} /$ $(\mathrm{kg} \mathrm{KG} \times \mathrm{d})$ bei Weibchen. Am Ende des Behandlungszeitraums war das Körpergewicht der Männchen in der höchsten Dosierung im Vergleich zur Kontrolle leicht vermindert. Das absolute und relative Lebergewicht war bei den Männ- chen ab 1200 ppm, bei den Weibchen bei 7200 ppm erhöht. Veränderungen des Gewichts anderer Organe zeigten keine Dosisabhängigkeit oder kein histologisches Korrelat und wurden daher von den Autoren nicht als substanzbedingt bewertet. In der Leber wurden vermehrt klarzellige, eosinophile und basophile Foci sowie eine zentrilobuläre Hypertrophie beobachtet. Außerdem war bei den Männchen die Zahl der Adenome und Karzinome, bei den Weibchen die der Adenome jeweils in der höchsten Dosierung signifikant erhöht [43] (NOAEL/LOAEL bei Männchen,Weibchen für nicht kanzerogene Effekte: 89/173 (M), 221/1399 mg/(kg $\mathrm{KG} \times \mathrm{d})(\mathrm{W}))$.

Die bei männlichen Mäusen im Vergleich zur mitgeführten Kontrollgruppe in allen Dosierungen schwach erhöhte Inzidenz von Adenomen + Adenokarzinomen der Lunge wird nicht als substanzbedingt gewertet, da die Inzidenz in der Kontrollgruppe sehr niedrig war, die Inzidenz in den anderen Gruppen im Bereich der historischen Kontrollen lag und die auch spontan bei diesem Mäusestamm auftretenden Tumoren in ihrer Inzidenz eine beträchtliche Variabilität zeigen [7].

Ergänzende Untersuchungen, in denen B6C3F1-Mäuse bis zu 4 Wochen 7200 ppm im Futter erhielten, haben gezeigt, dass NMP die Zellproliferation in der Leber steigert. Daraus wurde geschlossen, dass die nur bei der höchsten Dosis erhöhte Inzidenz von Lebertumoren bei diesem Mäusestamm auf eine nicht-gentoxische, zellproliferationsstimulierende Wirkung von NMP zurückgeführt werden kann $[7,44]$. 
Untersuchungen mit inhalativer Exposition Untersucht wurde die chronische Toxizität und Kanzerogenität von NMP bei inhalativer Exposition von Sprague-DawleyRatten [40]. Dazu wurden je 120 Männchen und Weibchen pro Gruppe bis zu 2 Jahre lang $6 \mathrm{~h} / \mathrm{d}, 5 \mathrm{~d} /$ Woche gegenüber 0 , 40 oder $400 \mathrm{mg} \mathrm{NMP} / \mathrm{m}^{3}$ ganzkörperexponiert. Die Exposition erfolgte gegenüber NMP-Dampf mit allenfalls Spuren von Aerosolanteilen. Bei der höchsten Konzentration war bei den männlichen Tieren die Gewichtszunahme signifikant leicht (6\%) vermindert und das Harnvolumen erhöht. Bei beiden Geschlechtern war bei der höchsten Konzentration der Urin dunkelgelb gefärbt, und in der Lunge fanden sich im numerischen Vergleich zur Kontrolle häufiger Anzeichen minimaler Veränderungen (Männchen: akute fokale Alveolitis bei 10/85 Tieren vs. 2/84 Tieren in der Kontrolle, außerdem Hyperplasie der Alveolarzellen bei 4/84 vs. 0/84 Kontroll-Tieren; Weibchen: Alveolarzellhyperplasie bei 9/84 Tieren vs. 4/84 in der Kontrolle). Weibchen beider und Männchen der höheren Dosierung zeigten häufiger gefärbte, feuchte Dammgegenden als die Kontrolltiere. In einer nach 18 Monaten untersuchten Teilgruppe von Männchen waren der Hämatokrit und die Aktivität der Alkalischen Phosphatase im Serum erhöht, diese Effekte waren nach 2 Jahren nicht mehr nachweisbar. Männliche Ratten in der Gruppe mit der höchsten Exposition, die vor Ablauf von 18 Monaten Versuchsdauer starben oder wegen schlechten Allgemeinzustands getötet wurden, zeigten häufiger als Kontrolltiere eine chronische Nephropathie mit Glomerulosklerose, die Unterschiede zwischen beiden Gruppen waren aber weder nach 18 noch nach 24 Monaten signifikant. Auch die Mortalität sowie die Inzidenz neoplastischer und anderer Organveränderungen war in den exponierten Gruppen im Vergleich zur Kontrollgruppe nicht erhöht (NOAEC: $40 \mathrm{mg} / \mathrm{m}^{3}$, LOAEC: $\left.400 \mathrm{mg} / \mathrm{m}^{3}\right)$.

\section{Untersuchungen mit dermaler Exposition} Es liegen keine Angaben vor.

\subsection{Wirkung auf Reproduktion und Entwicklung}

Befunde am Menschen liegen bis auf einen einzigen Fallbericht nicht vor. Dieser beschreibt den Fall einer Schwangeren, die in den ersten 20 Wochen ihrer Schwangerschaft beruflich gegenüber NMP exponiert war, vermutlich in erster Linie durch Hautkontakt mit flüssigem NMP. In der 16. Woche der Schwangerschaft kam es zu einer Kontamination von Kleidung und Haut durch Verschütten von NMP. Über die Höhe der Exposition liegt keine Abschätzung vor. In der Folge klagte die Betroffene über Kopfschmerzen, Übelkeit und allgemeines Krankheitsgefühl. In der 25. Woche wurde eine verzögerte Embryonalentwicklung festgestellt, in der 31. Woche erfolgte nach einer anfänglich unauffälligen Schwangerschaft eine Totgeburt $[15,45]$.

Die tierexperimentellen Studien, die Effekte von NMP auf die Reproduktion und die embryonale Entwicklung untersucht haben, sind in den folgenden Kapiteln dargestellt.

\subsubsection{Reproduktionstoxizität Untersuchungen mit oraler Exposition}

In einer nur zusammenfassend vom RIVM [13] beschriebenen 2-Generationenstudie an Sprague-Dawley-Ratten (je 30 Männchen und Weibchen/Dosis) erhielten die Tiere 0, 50, 160 oder $500 \mathrm{mg}$ $\mathrm{NMP} /(\mathrm{kg} \mathrm{KG} \times \mathrm{d})$ mit dem Futter [46] Nach Auffassung der Autoren waren adverse Wirkungen auf Reproduktion und Fertilität sowie auf die Überlebensrate von Jungtieren nur bei der höchsten Dosierung feststellbar, die bereits parental toxisch wirkte. Veränderungen bei niedrigeren Dosierungen lagen noch im Bereich historischer Kontrollen. Hingegen vertrat die US-amerikanische Umweltbehörde EPA die Auffassung, dass auch die Minderung der männlichen Fertilität und der weiblichen Fruchtbarkeit bei den beiden niedrigeren Dosierungen als biologisch bedeutsam anzusehen seien, auch wenn die Veränderungen noch nicht statistisch signifikant waren.

Wegen der unklaren Bewertung wurden weitere 2-Generationen-Studien durchgeführt. In einer Studie an Wistar-Ratten [47] erhielten je 25 Männchen und Weibchen ab 10 Wochen vor der Verpaarung und während der Verpaarung, Trächtigkeit, Laktation und einer Ruhephase zwischen den Trächtigkeiten mit dem Futter fortlaufend adjustierte Mengen an NMP, die zugeführte Dosen von 0, 50, 160 oder $500 \mathrm{mg} /(\mathrm{kg} \mathrm{KG} \times \mathrm{d})$ ergaben. Bei den Jungtieren des ersten Wurfs (F1a) unter der höchsten Dosierung war eine hohe Mortalität an den postnatalen Tagen 1-4 zu verzeichnen, die auf pränatale entwicklungstoxische Effekte zurückgeführt wurde. Wegen dieser hohen Mortalität wurde die höchste Dosierung im weiteren Studienverlauf auf $350 \mathrm{mg} /(\mathrm{kg} \mathrm{KG} \times$ d) reduziert. NMP hatte keinen behandlungsbedingten Effekt auf den Reproduktionserfolg oder die Fertilität der F0und F1-Elterntiere. Auch die abgesenkte höchste Dosis von $350 \mathrm{mg} /(\mathrm{kg} \mathrm{KG} \times$ d) führte zu Anzeichen systemischer Toxizität mit verminderter Gewichtszunahme und Futteraufnahme sowie Nierenveränderungen bei den Elterntieren und entwicklungstoxischen Wirkungen (erhöhte Mortalität, verminderte Gewichtszunahme) bei den Jungtieren (detaillierte Angaben nicht verfügbar). In dieser Untersuchung lag der NOAEL für systemische und Entwicklungs-Toxizität bei $160 \mathrm{mg} /$ $(\mathrm{kg} \mathrm{KG} \times \mathrm{d})($ LOAEL: $350 \mathrm{mg} /(\mathrm{kg} \mathrm{KG} \times$ d)).

In einer entsprechend konzeptionierten zweiten Studie an Sprague-DawleyRatten [48] war ebenfalls bei der anfänglich höchsten Dosierung von $500 \mathrm{mg} /(\mathrm{kg}$ $\mathrm{KG} \times \mathrm{d}$ ) postnatal (Laktationstag 1-4) die Mortalität der Jungtiere erhöht, was wiederum auf pränatale entwicklungstoxische Effekte zurückgeführt wurde. Auch hier hatte NMP keinen Einfluss auf die Fertilität. Die abgesenkte Dosis von $350 \mathrm{mg} /(\mathrm{kg} \mathrm{KG} \times \mathrm{d})$ ließ außerdem im Unterschied zur Studie an Wistar-Ratten keine behandlungsbedingten systemischtoxischen Wirkungen erkennen, führte jedoch bei den F2b-Nachkommen zu vermindertem Körpergewicht und einer verminderten Zahl an Jungtieren, die die Laktationsphase überlebten. In dieser Studie lag der NOAEL für Fertilität und systemische Toxizität bei $350 \mathrm{mg} /(\mathrm{kg} \mathrm{KG} \times$ d), der NOAEL für Entwicklungstoxizität bei $160 \mathrm{mg} /(\mathrm{kg} \mathrm{KG} \times \mathrm{d})(\mathrm{LOAEL}: 350 \mathrm{mg} /$ $(\mathrm{kg} K G \times d))$. 


\begin{tabular}{|c|c|c|c|c|}
\hline \multirow{2}{*}{ Parameter/Effekt } & \multicolumn{4}{|c|}{ Orale Dosis $(\mathrm{mg} /(\mathrm{kg} \mathrm{KG} \times \mathrm{d}))$} \\
\hline & 0 & 150 & 450 & 1000 \\
\hline $\begin{array}{l}\text { Körpergewicht (g) der Weibchen, Tag } \\
20 \text { der Gestation }\end{array}$ & $321,3 \pm 29,0^{a}(22)$ & $299,6 \pm 11,1(24)$ & $284,3 \pm 40,4(20)$ & $259,7 \pm 17,9(15)$ \\
\hline Anzahl verpaarter Weibchen & 24 & 26 & 28 & 22 \\
\hline $\begin{array}{l}\text { Anzahl lebender/ } \\
\text { toter Jungtiere pro Wurf }\end{array}$ & $\begin{array}{l}11,5 \pm 3,5 / \\
0,18 \pm 0,85\end{array}$ & $\begin{array}{l}10,4 \pm 2,6 \\
0\end{array}$ & $\begin{array}{l}10,5 \pm 3,4 \\
0,13 \pm 0,34\end{array}$ & $\begin{array}{l}0,33 \pm 0,82 \\
0,80 \pm 1,1\end{array}$ \\
\hline Fertilitätsindex & 91,7 & 92,3 & 71,4 & 68,2 \\
\hline Vitalitätsindex & 94,0 & 86,4 & 71,6 & 0 \\
\hline Laktationsindex & 96,1 & 78,2 & 43,4 & 0 \\
\hline $\begin{array}{l}\text { Körpergewicht (g) der Nachkommen, } \\
\text { postnatal Tag 4, Weibchen/Männchen }\end{array}$ & $\begin{array}{l}9,79 \pm 1,42(22) \\
11,05 \pm 1,13(22)\end{array}$ & $\begin{array}{l}7,35 \pm 1,20(24) \\
7,70 \pm 1,29(24)\end{array}$ & $\begin{array}{l}6,07 \pm 0,83(20) \\
6,34 \pm 1,02(20)\end{array}$ & - \\
\hline
\end{tabular}

Tab. 7 Zusammenstellung der NOAEL und LOAEL aus Fertilitätsstudien mit oraler Verabreichung von NMP

\begin{tabular}{|c|c|c|c|c|c|}
\hline $\begin{array}{l}\text { Spezies, Stamm, Anzahl } \\
\text { und Geschlecht/Dosis }\end{array}$ & Exposition & $\begin{array}{l}\text { NOAEL } \\
(\mathrm{mg} /(\mathrm{kg} \mathrm{KG} \times \mathrm{d}))\end{array}$ & $\begin{array}{l}\text { LOAEL } \\
(\mathrm{mg} /(\mathrm{kg} \mathrm{KG} \times \mathrm{d}))\end{array}$ & Effekt, Veränderungen beim LOAEL & Quelle \\
\hline Ratte, Wistar, 25 M/25 W & $\begin{array}{l}\text { 2-Generationen- } \\
\text { Studie }\end{array}$ & $\begin{array}{l}160 \text { (systemisch, Ent- } \\
\text { wicklung) } \\
350 \text { (Fertilität) }\end{array}$ & $\begin{array}{l}350 \text { (systemisch und } \\
\text { Entwicklung) }\end{array}$ & $\begin{array}{l}\text { Gewichtszunahme vermindert, Nie- } \\
\text { renveränderungen, Jungtiere: Morta- } \\
\text { lität, Gewichtszunahme vermindert }\end{array}$ & [47] \\
\hline $\begin{array}{l}\text { Ratte, Sprague-Dawley, } \\
25 \mathrm{M} / 25 \mathrm{~W}\end{array}$ & $\begin{array}{l}\text { 2-Generationen- } \\
\text { Studie }\end{array}$ & $\begin{array}{l}350 \text { (systemisch, Fertilität) } \\
160 \text { (Entwicklung) }\end{array}$ & $\begin{array}{l}\text {-(Systemisch, Fertili- } \\
\text { tät), } 350 \text { (Entwicklung) }\end{array}$ & $\begin{array}{l}\text { Jungtiere: Mortalität, Gewichtszu- } \\
\text { nahme vermindert }\end{array}$ & [48] \\
\hline Ratte, Wistar, 22-24 M & $\begin{array}{l}10 \text { Wochen, } 5 \mathrm{~d} / \\
\text { Woche vor Ver- } \\
\text { paarung }\end{array}$ & $\begin{array}{l}300 \text { (Fertilität) } \\
100 \text { (Entwicklung) }\end{array}$ & $\begin{array}{l}1000 \text { (Fertilität) } \\
300 \text { (Entwicklung) }\end{array}$ & $\begin{array}{l}\text { Hodenschäden, Fertilität vermindert, } \\
\text { Jungtiere: postnatal Mortalität }\end{array}$ & [49] \\
\hline Ratte, Wistar, 22-28 W & $\begin{array}{l}\text { Ab } 2 \text { Wochen vor } \\
\text { Verpaarung bis En- } \\
\text { de Laktation, } 5 \mathrm{~d} / \\
\text { Woche }\end{array}$ & - & 150 (niedrigste Dosis) & $\begin{array}{l}\text { W: Körpergewicht ab GD } 6 \text { ver- } \\
\text { mindert, Jungtiere: Überlebensrate } \\
\text { vermindert }\end{array}$ & [50] \\
\hline
\end{tabular}

Auswirkungen von NMP auf die Fertilität männlicher Wistar-Ratten und deren Nachkommen wurden von Sitarek \& Stetkiewicz [49] untersucht. Dazu erhielten sieben Wochen alte Männchen (2224 Tiere/Dosis) an $5 \mathrm{~d} /$ Woche 10 Wochen lang per Schlundsonde 0, 100, 300 oder $1000 \mathrm{mg} \mathrm{NMP} /(\mathrm{kg} \mathrm{KG} \times \mathrm{d})$. Anschließend wurden die Männchen mit unbehandelten Weibchen verpaart und Überleben und Entwicklung der ausgetragenen Jungtiere bis zum 28. Tag postnatal verfolgt. In der höchsten Dosis verursachte NMP ausgeprägte Schäden im Keimepithel der Hoden und verminderte die Fertilität der Männchen. Nur zwei der 44 Weibchen, die mit so behandelten Männchen verpaart worden waren, trugen (insgesamt sechs) Nachkommen aus. Unter den Nachkommen der Männchen, die mit $300 \mathrm{mg} /(\mathrm{kg} \mathrm{KG} \times \mathrm{d})$ behandelt worden waren, war postnatal die Überlebensrate bis zum Tag 4 reduziert. $100 \mathrm{mg} /(\mathrm{kg}$ $\mathrm{KG} \times \mathrm{d}$ ) hatten keinen Effekt auf Fertilität und Spermatogenese der Männchen oder die Entwicklung der Nachkommen. (NOAEL/LOAEL für Fertilität: 300/1000 mg/ $(\mathrm{kg} \mathrm{KG} \times \mathrm{d})$, für Entwicklungstoxizität 100/300 mg/(kg KG × d)).

In einer weiteren Untersuchung derselben Arbeitsgruppe [50] wurden Auswirkungen von NMP auf die Fertilität von weiblichen Wistar-Ratten (22-28 Tiere/ Dosis) untersucht (• Tab. 6). Dazu erhielten die Tiere an $5 \mathrm{~d}$ /Woche ab zwei Wochen vor der Verpaarung mit unbehandelten Männchen sowie während der Verpaarung und Trächtigkeit bis zum Ende der Laktation per Schlundsonde 0, 150, 450 oder $1000 \mathrm{mg} \mathrm{NMP} /(\mathrm{kg} \mathrm{KG} \times \mathrm{d})$. Bei der höchsten Dosis starben zwei der (noch nicht trächtigen) Weibchen am 30. bzw. 32. Tag der Behandlung. Ab der Dosis von $450 \mathrm{mg} /(\mathrm{kg} \mathrm{KG} \times \mathrm{d})$ war der Fertilitätsindex erniedrigt. In der höchsten Dosierung war die Zahl lebender Jungtiere signifikant vermindert und die Zahl an Totgeburten pro Wurf erhöht. Bei al- len Dosierungen waren die Überlebensrate der Jungtiere über die Gesamtphase von drei Wochen nach der Geburt vermindert und das Körpergewicht der Muttertiere ab dem 6. Tag der Gestation signifikant erniedrigt. Aus dieser Studie lässt sich kein NOAEL ableiten, da bereits die niedrigste Dosis adverse Wirkungen zeigte (LOAEL: $150 \mathrm{mg} /(\mathrm{kg} \mathrm{KG} \times \mathrm{d}) \bullet$ Tab. 7 gibt eine Übersicht über die Studien zur Reproduktionstoxizität von NMP nach oraler Verabreichung.)

Untersuchungen mit inhalativer Exposition Im Tierversuch wurde bei männlichen Ratten nach Ganzkörperexposition über 90 Tage $(6 \mathrm{~h} / \mathrm{d}) \mathrm{mit} 618 \mathrm{mg} / \mathrm{m}^{3} \mathrm{NMP}$ Dampf keine Veränderung des Hodengewichts, und -gewebes oder der Morphologie und Zahl der Spermien festgestellt [51]. In anderen Untersuchungen traten jedoch bei höherer Exposition mit Aerosol-Dampf-Gemischen (4 d bei $7000 \mathrm{mg}$ / $\mathrm{m}^{3}, 2$ Wochen bei $4000 \mathrm{mg} / \mathrm{m}^{3}$ oder 13 
Tab. 8 Zusammenstellung der NOAEC und LOAEC aus einer Fertilitätsstudie mit inhalativer Exposition gegenüber NMP

\begin{tabular}{|c|c|c|c|c|c|}
\hline $\begin{array}{l}\text { Spezies, Stamm, Anzahl } \\
\text { und Geschlecht/Dosis }\end{array}$ & Exposition & $\begin{array}{l}\text { NOAEC } \\
\left(\mathrm{mg} / \mathrm{m}^{3}\right)\end{array}$ & $\begin{array}{l}\text { LOAEC } \\
\left(\mathrm{mg} / \mathrm{m}^{3}\right)\end{array}$ & $\begin{array}{l}\text { Effekt, Veränderungen } \\
\text { beim LOAEC }\end{array}$ & Quelle \\
\hline $\begin{array}{l}\text { Ratte, Sprague-Dawley, } \\
10 \mathrm{M} / \\
20 \mathrm{~W}\end{array}$ & $\begin{array}{l}\text { 2-Generationen-Studie, } \\
6 \mathrm{~h} / \mathrm{d}, 5 \mathrm{~d} / \text { Woche, mind. } 14 \\
\text { Wochen, Ganzkörper }\end{array}$ & $\begin{array}{l}206 \text { (Entwicklung) } \\
478 \text { (Fertilität) }\end{array}$ & $\begin{array}{l}478 \text { (Entwicklung) } \\
\text { - (Fertilität) }\end{array}$ & $\begin{array}{l}\text { Jungtiere: Körpergewicht } \\
\text { bis zum 21. Tag postnatal } \\
\text { vermindert }\end{array}$ & [52] \\
\hline
\end{tabular}

Tab. 9 Zusammenstellung der NOAEL und LOAEL aus Studien zur Entwicklungstoxizität mit oraler Verabreichung von NMP

\begin{tabular}{|lclllc}
$\begin{array}{l}\text { Spezies, Stamm, Anzahl } \\
\text { und Geschlecht/Dosis }\end{array}$ & Exposition & $\begin{array}{l}\text { NOAEL } \\
(\mathbf{m g} /(\mathbf{k g ~ K G} \times \mathrm{d}))\end{array}$ & $\begin{array}{l}\text { LOAEL } \\
(\mathbf{m g} /(\mathbf{k g ~ K G} \times \mathbf{d}))\end{array}$ & Effekt, Veränderungen beim LOAEL \\
\hline $\begin{array}{l}\text { Ratte, Sprague-Dawley, } \\
\text { 21-25W }\end{array}$ & GD 6-20 & $\begin{array}{l}125 \text { (maternal und } \\
\text { Entwicklung) }\end{array}$ & $\begin{array}{l}250 \text { (maternal und } \\
\text { Entwicklung) }\end{array}$ & $\begin{array}{l}\text { Gewichtszunahme vermindert (maternal und } \\
\text { Jungtiere), Fehlbildungen }\end{array}$ \\
\hline $\begin{array}{llll}\text { Ratte, Sprague-Dawley, } \\
\text { 25W }\end{array}$ & GD 6-15 & $\begin{array}{l}125 \text { (maternal und } \\
\text { Entwicklung) }\end{array}$ & $\begin{array}{l}400 \text { (maternal und } \\
\text { Entwicklung) }\end{array}$ & $\begin{array}{l}\text { Gewichtszunahme vermindert (maternal und } \\
\text { Jungtiere) }\end{array}$ \\
\hline $\begin{array}{l}\text { Kaninchen, New Zealand, } \\
\text { 20W }\end{array}$ & GD 6-18 & $\begin{array}{l}55 \text { (maternal) } \\
175 \text { (Entwicklung) }\end{array}$ & $\begin{array}{l}175 \text { (maternal) } \\
540 \text { (Entwicklung) }\end{array}$ & $\begin{array}{l}\text { Gewichtszunahme vermindert, Entwicklung: } \\
\text { Postimplantationsverluste, Fehlbildungen }\end{array}$ \\
\hline
\end{tabular}

Wochen bei $3000 \mathrm{mg} / \mathrm{m}^{3}$ ) vermindertes Hodengewicht, histopathologische Veränderungen und Zellverlust im Keimepithel des Hodens auf [5].

In einer 2-Generationenstudie wurden je 10 männliche und 20 weibliche Sprague-Dawley-Ratten pro Dosis für mindestens 14 Wochen $6 \mathrm{~h} / \mathrm{d}, 5 \mathrm{~d} /$ Woche gegenüber $0,41,206$ oder $478 \mathrm{mg} \mathrm{NMP} / \mathrm{m}^{3}$ ganzkörperexponiert, und anschließend jeweils mit derselben Dosis exponierte Männchen und Weibchen verpaart [52]

(- Tab. 8). Ein Teil der Nachkommen ( $\mathrm{F}_{1}$-Generation), die ab dem 4 . Tag nach der Geburt exponiert wurden, wurde wie beschrieben gegenüber 0 oder $478 \mathrm{mg} / \mathrm{m}^{3}$ exponiert, und jeweils NMP-exponierte Tiere des einen Geschlechts mit Kontrolltieren des anderen für die $\mathrm{F}_{2}$-Generation verpaart. Es wurden keine Wirkungen auf Körpergewicht und Gewicht der Fortpflanzungsorgane oder die Reproduktionsfähigkeit festgestellt. Bei den Jungtieren der $\mathrm{F}_{1}$-Generation war das Körpergewicht bis zum 21. Tag, aber nicht am 28. Tag, um 4-11\% vermindert. Der Effekt zeigte im Trendtest bis auf die höchste Dosierung keine Dosisabhängigkeit. Für den Untersuchungsteil zur Entwicklungstoxizität erfolgte die Exposition wie beschrieben gegenüber 0 oder $478 \mathrm{mg} /$ $\mathrm{m}^{3}$, die trächtigen Tiere wurden am 21 . Tag schnittentbunden. In der Gruppe der exponierten Weibchen war das Körpergewicht der Föten im Vergleich zur Kontrollgruppe um $7 \%$ vermindert. Hinweise auf embryo-, fötotoxische oder teratogene Effekte ergaben sich nicht. Die Autoren der Studie leiten aus den Befunden eine NOAEC von $206 \mathrm{mg} / \mathrm{m}^{3}$ ab (LOAEC: $\left.478 \mathrm{mg} / \mathrm{m}^{3}\right)$.

\section{Untersuchungen mit dermaler Exposition}

Es liegen keine Angaben vor.

\subsubsection{Entwicklungstoxizität Untersuchungen mit oraler Exposition (•Tab. 9)}

In einer Untersuchung an weiblichen Sprague-Dawley-Ratten (21-25 trächtige Tiere/Dosis) erhielten die Tiere oral (Schlundsonde) vom 6.-20. Tag der Gestation 0, 125, 250, 500 oder 750 mg NMP/ $(\mathrm{kg} \mathrm{KG} \times \mathrm{d})$ [53]. Die maternale Gewichtszunahme bei $250 \mathrm{mg} /(\mathrm{kg} \mathrm{KG} \times \mathrm{d})$ war um $9 \%$ reduziert, eine vergleichbare Gewichtsminderung zeigten die Föten bei derselben Dosis. Das Nettogewicht der Muttertiere war ab $250 \mathrm{mg} /(\mathrm{kg} \mathrm{KG} \times \mathrm{d})$ mit steigender NMP-Dosis um 11, 26 und $26 \%$ reduziert. Das Fötusgewicht nahm dosisabhängig um 2,5-48\% ab, der Effekt war ab $250 \mathrm{mg} /(\mathrm{kg} \mathrm{KG} \times$ d) signifikant (10\% Gewichtsminderung). Ab $500 \mathrm{mg} /$ $(\mathrm{kg} \mathrm{KG} \times \mathrm{d})$ kamen weitere Effekte hinzu: verzögerte Verknöcherung des Schädels und der Sternebrae, vermehrtes Auftreten von Skelettvariationen und externen Weichteil- und Skelettfehlbildungen sowie vermehrt Postimplantationsverluste und Resorptionen. Ein einzelner Fötus wies bereits bei $250 \mathrm{mg} /(\mathrm{kg} \mathrm{KG} \times \mathrm{d})$ eine Fehlbildung (Nabelbruch) auf. Aus dieser Untersuchung ergibt sich ein NOAEL für maternale und entwicklungstoxische Effekte von $125 \mathrm{mg} /(\mathrm{kg} \mathrm{KG} \times \mathrm{d})$ (LOAEL: $250 \mathrm{mg} /(\mathrm{kg} \mathrm{KG} \times \mathrm{d})$ ).
Ergänzende vergleichende Untersuchungen derselben Arbeitsgruppe mit den drei NMP-Hauptmetaboliten 5-HNMP, NMSI und 2-HMSI ließen für 5-HNMP und 2-HMSI keine entwicklungstoxischen Wirkungen erkennen. NMSI zeigte ab $750 \mathrm{mg} /(\mathrm{kg} \mathrm{KG} \times \mathrm{d})$ maternal toxische, ab $1000 \mathrm{mg} /(\mathrm{kg} \mathrm{KG} \times \mathrm{d})$ teratogene und ab $1250 \mathrm{mg} /(\mathrm{kg} \mathrm{KG} \times \mathrm{d})$ embryotoxische Wirkungen. Die Metaboliten wirkten somit nicht oder erst in höheren Dosen als NMP selbst entwicklungstoxisch oder teratogen, sodass diese Wirkungen nicht auf diese Verbindungen zurückgeführt werden können [54].

In einer weiteren Studie an SpragueDawley-Ratten erhielten trächtige Weibchen (25/Dosis) vom 6.-15. Tag der Gestation $0,40,125$ oder $400 \mathrm{mg} \mathrm{NMP} /(\mathrm{kg}$ $\mathrm{KG} \times \mathrm{d}$ ) [55]. Die maternale Gewichtszunahme war ab $400 \mathrm{mg} /(\mathrm{kg} \mathrm{KG} \times \mathrm{d})$ in den Intervallen GD 6-9, 9-12 und 6-15 reduziert, nicht aber über die gesamte Gestationsphase (GD 0-21) und nach Korrektur um das Gewicht des graviden Uterus. Das Fötusgewicht war bei $125 \mathrm{mg} /(\mathrm{kg} \mathrm{KG} \times \mathrm{d})$ signifikant, jedoch gering (3\%) reduziert, bei $400 \mathrm{mg} /(\mathrm{kg} \mathrm{KG} \times \mathrm{d})$ war der Effekt ausgeprägt (10\%). Bei $400 \mathrm{mg} /(\mathrm{kg} \mathrm{KG} \times$ d) war die Zahl von Föten mit gehemmter Entwicklung erhöht, im Übrigen zeigten sich keine maternal oder entwicklungstoxischen Effekte oder teratogenen Wirkungen (NOAEL/LOAEL für maternale und Entwicklungstoxizität: 125/400 mg/ $(\mathrm{kg} K G \times d)$ ).

In einer unveröffentlichten Untersuchung an Kaninchen [56] erhielten je 20 trächtige Tiere (New Zealand White)/Do- 


\begin{tabular}{|c|c|c|c|c|c|}
\hline $\begin{array}{l}\text { Spezies, Stamm, Anzahl } \\
\text { und Geschlecht/Dosis }\end{array}$ & Exposition & $\begin{array}{l}\text { NOAEC } \\
\left(\mathrm{mg} / \mathrm{m}^{3}\right)\end{array}$ & $\begin{array}{l}\text { LOAEC } \\
\left(\mathrm{mg} / \mathrm{m}^{3}\right)\end{array}$ & Effekt, Veränderungen beim LOAEC & Quelle \\
\hline $\begin{array}{l}\text { Ratte, Sprague-Dawley, } \\
10 \mathrm{M} / 10 \mathrm{~W}\end{array}$ & $\begin{array}{l}\text { 2-Generationen-Studie, } \\
6 \mathrm{~h} / \mathrm{d}, 5 \mathrm{~d} / \text { Woche, mind. } \\
14 \text { Wochen, Ganzkörper }\end{array}$ & $\begin{array}{l}206 \text { (Entwicklung) } \\
478 \text { (Fertilität) }\end{array}$ & $\begin{array}{l}478 \text { (Entwicklung) } \\
\text { - (Fertilität) }\end{array}$ & $\begin{array}{l}\text { Jungtiere: Körpergewicht bis zum 21. Tag } \\
\text { postnatal vermindert }\end{array}$ & [52] \\
\hline $\begin{array}{l}\text { Ratte, Sprague-Dawley, } \\
25-26 \text { W }\end{array}$ & $\begin{array}{l}\text { GD 6-20, } 6 \mathrm{~h} / \mathrm{d} \text {, } \\
\text { Ganzkörper }\end{array}$ & $\begin{array}{l}124 \text { (maternal) } \\
247 \text { (Entwicklung) }\end{array}$ & $\begin{array}{l}247 \text { (maternal) } \\
494 \text { (Entwicklung) }\end{array}$ & $\begin{array}{l}\text { Gewichtszunahme vermindert, Entwick- } \\
\text { lung: Fötusgewicht vermindert }\end{array}$ & [57] \\
\hline $\begin{array}{l}\text { Ratte, Sprague-Dawley, } \\
25 \mathrm{~W}\end{array}$ & $\begin{array}{l}\text { GD 6-15, } 6 \text { h/d, } \\
\text { Ganzkörper }\end{array}$ & $\begin{array}{l}- \text { (maternal) } \\
360 \text { (Entwicklung) }\end{array}$ & $\begin{array}{l}100 \text { (maternal) } \\
- \text { (Entwicklung) }\end{array}$ & $\begin{array}{l}\text { GD 6-8 sporadisch Lethargie, unregel- } \\
\text { mäßige Atmung, ansonsten keine Effekte }\end{array}$ & [40] \\
\hline $\begin{array}{l}\text { Kaninchen, Himalaya, } \\
15 \mathrm{~W}\end{array}$ & $\begin{array}{l}\text { GD 7-19, } 6 \mathrm{~h} / \mathrm{d} \text {, } \\
\text { Ganzkörper }\end{array}$ & $\begin{array}{l}500 \text { (maternal und } \\
\text { Entwicklung) }\end{array}$ & $\begin{array}{l}1000 \text { (maternal } \\
\text { und Entwicklung) }\end{array}$ & $\begin{array}{l}\text { Hepatotoxizität, Entwicklung: vermehrt } \\
\text { Skelettvariationen und Resorptionen }\end{array}$ & {$[58,59]$} \\
\hline
\end{tabular}

sis vom 6.-18. Tag der Gestation wässrige NMP-Lösungen $(0,55,175,540 \mathrm{mg} /(\mathrm{kg}$ $\mathrm{KG} \times \mathrm{d})) . \mathrm{Ab} 175 \mathrm{mg} /(\mathrm{kg} \mathrm{KG} \times \mathrm{d})$ traten maternal toxische Effekte auf (verminderte Gewichtszunahme). Bei der höchsten Dosierung traten vermehrt Postimplantationsverluste durch frühe und späte Resorptionen auf und die Wurfgrößen waren verringert. Außerdem zeigten sich Fehlbildungen des kardiovaskulären Systems und des Skeletts. Detaillierte Ergebnisse dieser Studie sind nicht publiziert. Für diese Untersuchung wird für maternal toxische Effekte ein NOAEL/LOAEL von 55/175 mg/(kg KG × d), für entwicklungstoxische sowie teratogene Effekte von 175/540 mg/(kg KG × d) angegeben.

\section{Untersuchungen mit inhalativer Exposition (• Tab. 10)}

Im Rahmen einer 2-Generationenstudie mit durchgeführte Untersuchungen zur Entwicklungstoxizität sind im vorhergehenden Kapitel bei dieser Studie [52] beschrieben.

In einer Studie zur Entwicklungstoxizität wurden trächtige Sprague-DawleyRatten (25-26/Dosis) vom 6.-20. Tag der Trächtigkeit gegenüber $0,124,247$ oder $494 \mathrm{mg} / \mathrm{m}^{3}$ aerosolfreiem NMP $6 \mathrm{~h} / \mathrm{d}$ ganzkörperexponiert [57]. Bei den Muttertieren war die Gewichtszunahme der NMP-exponierten Tiere im Mittel geringer als in der Kontrollgruppe, der Effekt war vom 6.-13. Tag der Gestation ab der mittleren Dosis signifikant. Wurde die Gewichtsveränderung um das Gewicht des graviden Uterus korrigiert, zeigte sich kein derartiger Effekt. In der höchsten Dosierung war außerdem der Futterverbrauch der Tiere reduziert und das Gewicht der Föten signifikant geringfügig (5\%) vermindert. Andere embryo- oder fötotoxische oder teratogene Wirkungen traten nicht auf (NOAEC/LOAEC für maternale Effekte: 124 bzw. $247 \mathrm{mg} / \mathrm{m}^{3}$; für entwicklungstoxische Effekte 247 bzw. $494 \mathrm{mg} / \mathrm{m}^{3}$ ).

In einer weiteren Studie zur Entwicklungstoxizität wurde nach Ganzkörperexposition trächtiger Sprague-DawleyRatten (25 Tiere/Dosis) mit 0, 100 oder $360 \mathrm{mg} / \mathrm{m}^{3}$ eines Dampf-Aerosolgemisches, 6 h/d, vom 6.-15. Tag der Trächtigkeit bei einigen exponierten Tieren in den ersten drei Tagen sporadisch Lethargie und unregelmäßige Atmung beobachtet. Weitere maternal toxische Effekte, Beeinträchtigungen der Embryonalentwicklung oder teratogene Effekte traten nicht auf [40]. Bei höheren Dosierungen wurden in anderen Untersuchungen jedoch entwicklungstoxische Wirkungen beobachtet: So führte Ganzkörperexposition von Ratten bei $680 \mathrm{mg} / \mathrm{m}^{3}$ zu erhöhten Resorptionsraten, vermindertem Fötusgewicht und verzögerter Verknöcherung und bei den Nachkommen exponierter Tiere bei $622 \mathrm{mg} / \mathrm{m}^{3}$ zu verzögerter Entwicklung [51].

Entwicklungstoxische Effekte wurden auch bei Kaninchen beobachtet. In einer unveröffentlichten Untersuchung [14, $58,59]$ wurden trächtige Weibchen (Himalaya, 15/Dosis) gegenüber 0, $200 \mathrm{mg} /$ $\mathrm{m}^{3}$ (nur Dampf), 500 bzw. $1000 \mathrm{mg} / \mathrm{m}^{3}$ (Dampf-Aerosolgemisch) an $6 \mathrm{~h} / \mathrm{d}$ vom 7.-19. Tag der Trächtigkeit exponiert und am 29. Tag schnittentbunden. Bei $1000 \mathrm{mg} / \mathrm{m}^{3}$ traten vermehrt Skelettvariationen (zusätzliche 13. Rippe) auf, jedoch keine teratogenen Wirkungen. Maternale Toxizität wurde in dieser Studie nicht festgestellt, machte sich aber in einer vorgeschalteten Dosisfindungsstudie bei 1000 und $2000 \mathrm{mg} / \mathrm{m}^{3}$ durch hepatotoxi- sche Effekte (erhöhtes Lebergewicht und Beeinträchtigungen klinisch-chemischer Parameter der Leberfunktion) bemerkbar. Die höchste Konzentration führte in dieser Vorstudie außerdem vermehrt zu Resorptionen und verminderte die Zahl lebender Föten. Die Versuchsanordnung wurde so gewählt, dass eine zusätzliche orale Aufnahme durch Putzen des Fells unwahrscheinlich ist, jedoch kann eine dermale Aufnahme nicht ausgeschlossen werden. (NOAEC/LOAEC für maternale und entwicklungstoxische Effekte: $500 \mathrm{mg} / \mathrm{m}^{3} / 1000 \mathrm{mg} / \mathrm{m}^{3}$ ).

\section{Untersuchungen mit dermaler Exposition (• Tab.11)}

Je 25 Sprague-Dawley-Ratten wurden vom 6.-15. Tag der Gestation mit 0, 75, 237 oder $750 \mathrm{mg} /(\mathrm{kg} \mathrm{KG} \times \mathrm{d}) \mathrm{NMP}$ behandelt $[60,61]$. Die Testsubstanz wurde dazu als wässrige Lösung auf die Haut (25 $\mathrm{cm}^{2}$ Fläche) aufgebracht, eingerieben und nach 8 h abgewaschen. Ein Kragen verhinderte die orale Aufnahme. Die höchste Dosierung wirkte deutlich maternal toxisch (um $28 \%$ verminderte Gewichtszunahme), hautreizend sowie entwicklungstoxisch und teratogen (verminderte Zahl lebender Föten, erhöhte Inzidenz von Resorptionen, vermindertes Fötusgewicht, verzögerte Skelettentwicklung, Fehlbildungen des Skeletts) (NOAEL/LOAEL für maternale und entwicklungstoxische Effekte 237/750 mg/(kg KG× d)).

In einer weiteren Studie wurden trächtige Kaninchen (weiße Himalaya, je 15/ Dosis) vom 7.-19. Tag der Gestation jeweils 6 h/d unter semiokklusiver Abdeckung der Haut gegen NMP-Dosen (40\% in Wasser) von $0,100,300$ oder 1000 $\mathrm{mg} \mathrm{NMP/(kg} \mathrm{KG \times d)} \mathrm{exponiert} \mathrm{[62].}$ Eine Gelbfärbung des Urins der Mutter- 
Tab. 11 Zusammenstellung der NOAEL und LOAEL aus Studien zur Entwicklungstoxizität mit dermaler Verabreichung von NMP

\begin{tabular}{|c|c|c|c|c|c|}
\hline $\begin{array}{l}\text { Spezies, Stamm, Anzahl } \\
\text { und Geschlecht/Dosis }\end{array}$ & Exposition & $\begin{array}{l}\text { NOAEL } \\
(\mathrm{mg} /(\mathrm{kg} \mathrm{KG} \times \mathrm{d}))\end{array}$ & $\begin{array}{l}\text { LOAEL } \\
(\mathrm{mg} /(\mathrm{kg} \mathrm{KG} \times \mathrm{d}))\end{array}$ & Effekt, Veränderungen beim LOAEL & Quelle \\
\hline $\begin{array}{l}\text { Ratte, Sprague-Dawley, } \\
25 \text { W }\end{array}$ & $\begin{array}{l}\text { GD 6-15, } \\
(8 \mathrm{~h} / \mathrm{d})\end{array}$ & $\begin{array}{l}237 \text { (maternal und } \\
\text { Entwicklung) }\end{array}$ & $\begin{array}{l}750 \text { (maternal und } \\
\text { Entwicklung) }\end{array}$ & $\begin{array}{l}\text { Gewichtszunahme vermindert, Entwicklung: föto- } \\
\text { toxisch, Skelettvariationen und -fehlbildungen }\end{array}$ & {$[60,61]$} \\
\hline $\begin{array}{l}\text { Kaninchen, Himalaya, } \\
15 \text { W }\end{array}$ & GD 7-19 & $\begin{array}{l}1000 \text { (maternal) } \\
300 \text { (Entwicklung) }\end{array}$ & $\begin{array}{l}\text { - (maternal) } \\
1000 \text { (Entwicklung) }\end{array}$ & Skelettvariationen & [62] \\
\hline
\end{tabular}

tiere ließ erkennen, dass die Testsubstanz systemisch verfügbar war. Maternal toxische Wirkungen traten nicht auf. Bei den Nachkommen wurden keine Fehlbildungen induziert, jedoch war eine leichte $\mathrm{Zu}$ nahme von Variationen wie überzählige 13. Rippen, Wirbelbögen und vorzeitige Verknöcherung des Sprungbeins zu verzeichnen, die als behandlungsbedingt betrachtet wurde (NOAEL für maternale Toxizität: $1000 \mathrm{mg} /(\mathrm{kg} \mathrm{KG} \times \mathrm{d})$, NOAEL/LOAEL für Entwicklungstoxizität: 300/1000 mg/(kg KG × d)).

\subsection{Sonstige Effekte}

\subsubsection{Neurotoxizität}

Die Ergebnisse einer kombinierten oralen subchronischen Studie/Neurotoxizitätsstudie sind im Kapitel. 4.5 beschrieben.

\subsubsection{Immuntoxizität}

Untersuchungen zur immuntoxischen Wirkung von NMP liegen nicht vor.

In einer Studie wurden jedoch Auswirkungen einer inhalativen NMP-Exposition auf immunologische Reaktionen an einem Maus-Modell beschrieben. In der betreffenden Studie [1] wurde an Mäusen, die gegenüber Ovalbumin sensibilisiert worden waren, die Wirkung einer inhalativen Exposition gegenüber NMP auf die allergische Reaktion gegen Ovalbumin untersucht. Um eine akute allergische Entzündung der Atemwege hervorzurufen, erhielten weibliche BALB/cByJMäuse am 1. und 14. Tag der Studie jeweils i.p. $20 \mu \mathrm{g}$ Ovalbumin auf Al-/Mghydroxid und am 14. sowie 17.-19. Tag je $40 \mu$ Ovalbumin in Salzlösung intranasal verabreicht. Mindestens 9 Tiere wurden $5 \mathrm{~h} / \mathrm{d}$ von Tag 0-19 oder nur vom 17.-19. Tag gegenüber der Umgebungsluft oder Umgebungsluft mit zugesetztem NMP $\left(0,019\right.$ oder $\left.0,051 \mathrm{mg} \mathrm{NMP} / \mathrm{m}^{3}\right)$ ganzkörperexponiert. Wurden die Tiere während der Sensibilisierung gegen Ovalbumin mit $0,019 \mathrm{mg} \mathrm{NMP} / \mathrm{m}^{3}$ exponiert, so zeig- te sich im Vergleich zur Kontrollgruppe eine verstärkte Entzündungsreaktion in der Lunge mit erhöhter Zahl eosinophiler Granulozyten in der bronchoalveolären Lavage (BAL), erhöhtem Th2-Cytokin und antigenspezifischem IgE-Spiegel bei erniedrigter Bildung von $\gamma$-Interferon. Der unter Methacholinprovokation gemessene Lungenwiderstand war jedoch im Vergleich zur Kontrolle nicht erhöht. Auf die stattfindende allergische Entzündung in bereits sensibilisierten Mäusen hatten $0,019 \mathrm{mg} \mathrm{NMP} / \mathrm{m}^{3}$ keinen Einfluss, bei $0,051 \mathrm{mg} / \mathrm{m}^{3}$ waren jedoch auch hier erhöhte antigenspezifische IgE-Spiegel und Th2-Cytokingehalte sowie eine verstärkte eosinophile Entzündungsreaktion in der BAL feststellbar. Ähnliche Veränderungen wurden auch beobachtet, wenn die Tiere den gasförmigen Ausdünstungen von Teppichboden ausgesetzt wurden, die neben Substanzen wie 2,2,4-Trimethyl-1,3-pentan-diol-diisobutyrat (TXIB), Glykolethern, 2-Ethyl-1-hexanol, Aliphaten und Phenol einen hohen Anteil NMP enthielten. Die entzündlichen Veränderungen konnten durch gleichzeitige Gabe von N-Acetylcystein im Trinkwasser verhindert werden.

\section{Bewertungen}

Im Gefahrstoffrecht ist NMP gemäß Verordnung (EG) Nr. 1272/2008 (CLP-Verordnung) [63] als reproduktionstoxisch, Kategorie 1B, eingestuft und mit dem Gefahrenhinweis H360D („Kann das Kind im Mutterleib schädigen") gekennzeichnet. Weiterhin ist NMP als augenreizend (Kategorie 2, H319: „Verursacht schwere Augenreizung“), hautreizend (Kategorie 2, H315: „Verursacht Hautreizungen") sowie atemwegreizend (Kategorie STOT SE 3, H335: „Kann die Atemwege reizen") gekennzeichnet bzw. eingestuft [64]. NMP wurde in der EU aufgrund seiner reproduktionstoxischen Effekte in die Liste der „besonders besorgnis- erregenden Stoffe" (SVHC: substances of very high concern) aufgenommen $[16,65,66]$.

Auf Basis dieser Einstufung wurde zum Schutz von Beschäftigten (einschließlich Schwangeren) am Arbeitsplatz eine max. Konzentration (DNEL) von $5 \mathrm{mg} / \mathrm{m}^{3}$ für den inhalativen und eine max. Dosis von 2,4 mg/(kg KG $\times$ d) für den dermalen Expositionspfad vorgeschlagen [15]. Als Ausgangspunkt für die Bewertung (POD) wurde für den inhalativen Pfad eine NOAEC (Effekt: reduziertes Fötusgewicht) von $247 \mathrm{mg} / \mathrm{m}^{3}$ (umgerechnet auf Arbeitsplatzexposition: $124 \mathrm{mg} / \mathrm{m}^{3}$ ) aus einer reproduktionstoxischen Studie an Ratten herangezogen [57], für den dermalen Pfad ein NOAEL von $237 \mathrm{mg} /(\mathrm{kg} \mathrm{KG} \times \mathrm{d})$ (Effekt: reduzierte Zahl lebender Föten und vermindertes Fötusgewicht bei Ratten) [60, 61]. Extrapolationsfaktoren zur Inter- und Intraspeziesextrapolation wurden gemäß ECHA-Richtlinie angesetzt; allerdings wurde zum Schutz von Beschäftigten einschließlich schwangeren Beschäftigten ein Intraspeziesfaktor von 10 statt 5 gewählt. Für Beschäftigte (ohne Schwangere) wurde entsprechend mit einem Intraspeziesfaktor von 5 gemäß ECHA-Richtlinie ein DNEL von $10 \mathrm{mg} / \mathrm{m}^{3}$ bzw. 4,6 mg/(kg KG $\times d$ ) vorgeschlagen [15].

Das niederländische RIVM hat in seinem Vorschlag zur Begrenzung des Einsatzes von NMP keinen tolerablen Wert für die Allgemeinbevölkerung vorgelegt [15] und die Verwendung von NMP in Verbraucherprodukten nicht betrachtet, da zum einen wegen der Aufnahme von NMP in die SVHC-Liste von einer sinkenden Verwendung von NMP in Verbraucherprodukten ausgegangen wird. Zum anderen wird in einem weiteren Dossier des RIVM [16] vorgeschlagen, die Höchstgrenze von NMP, ab der Produkte als reproduktionstoxisch gekennzeichnet werden sollten, von 5 auf 0,3\% zu senken. Dies würde nach den Ausführungen des 
RIVM die Verwendung von NMP in Verbraucherprodukten praktisch ausschließen, da eine NMP-Konzentration unterhalb von $0,3 \%$ keinen funktionellen Einsatz mehr zuließe.

Die Weltgesundheitsorganisation hat im „Concise International Chemical Assessment Document" (CICAD) für NMP eine Konzentration von $0,3 \mathrm{mg} / \mathrm{m}^{3}$ bei inhalativer Exposition als tolerabel erachtet [13]. Dieser Wert basiert auf der NOAEC von $500 \mathrm{mg} / \mathrm{m}^{3}$ einer subchronischen Inhalationsstudie an Ratten, in der die Tiere („head only“) gegenüber einem AerosolDampfgemisch exponiert worden waren [41], und der Umrechnung auf kontinuierliche $(\times(6 / 24) \times(5 / 7))$ und chronische Exposition (Faktor 3) sowie Berücksichtigung der Intraspezies- (Faktor 10) und Interspeziesvariabilität (Faktor 10). Das wissenschaftliche Komitee für Verbrauchersicherheit der EU (SCCS) hat in seiner Bewertung von NMP [3] keine tolerable Dosis abgeleitet, zieht aber den NOAEL von $125 \mathrm{mg} /(\mathrm{kg} \mathrm{KG} \times \mathrm{d})$ aus der Studie zur Entwicklungstoxizität von Saillenfait et al. [53] sowie Exxon [55] zur Abschätzung der „Margin of Safety“ (MOS) heran. In der Bewertung des SCCS [3] konnte allerdings die erst später veröffentlichte Studie von Sitarek et al. [50] noch nicht berücksichtigt werden, in der ein LOAEL von $150 \mathrm{mg} /(\mathrm{kg} \mathrm{KG} \times \mathrm{d})$, jedoch kein NOAEL ermittelt wurde.

Die vorliegenden Befunde zur Gentoxizität lassen nicht auf mutagene oder gentoxische Wirkungen von NMP schließen. Zwei Kanzerogenitätsstudien mit inhalativer bzw. oraler Exposition ergaben bei Ratten keine Hinweise auf ein kanzerogenes Potential. Bei B6C3F1-Mäusen traten nach oraler Verabreichung hoher Dosen vermehrt Leberadenome und -adenokarzinome auf. Ergänzende Untersuchungen zum Wirkungsmechanismus weisen darauf hin, dass NMP in der Leber des eingesetzten Mäusestamms die Zellproliferation stimuliert. Angesichts der fehlenden Gentoxizität, der bekannten Empfindlichkeit des verwendeten Mäusestamms im Hinblick auf die Ausbildung von Lebertumoren, des Befunds, dass derartige Tumore nach NMP-Verabreichung nur bei der höchsten Dosierung vermehrt auftraten, und der proliferationsfördernden Wirkung von NMP in der Leber dieser Mäuse ist ein epigenetischer Mechanismus mit einem Schwellenwert wahrscheinlich. Für die Bewertung von NMP-Effekten im Niedrigdosisbereich zum Schutz der menschlichen Gesundheit werden diese Befunde an Mäusen daher nicht herangezogen.

In einer neueren tierexperimentellen Untersuchung an Mäusen, die gegenüber Ovalbumin sensibilisiert wurden, wird berichtet, dass die durch Ovalbuminprovokation hervorgerufene entzündliche allergische Reaktion der Atemwege durch inhalative Exposition gegenüber NMP verstärkt wird. Dabei lagen die eingesetzten wirksamen Konzentrationen von NMP unter $0,1 \mathrm{mg} / \mathrm{m}^{3}$ und somit um mehrere Größenordnungen unter denen anderer Studien. Für eine sensibilisierende Wirkung von NMP selbst liegen weder aus Tierversuchen noch aus Befunden beim Menschen Hinweise vor. Die Relevanz dieser Befunde für den Menschen ist gegenwärtig unbekannt. Insbesondere ist unklar, ob bei Personen mit bestehender Sensibilisierung der Atemwege gegenüber anderen Stoffen tatsächlich adverse Veränderungen durch NMP hervorgerufen werden können. Hierzu liegen bisher keine Hinweise vor [67]. Aus diesen Gründen können die genannten Befunde nicht für die Bewertung von NMP herangezogen werden.

\section{Ableitung von HBM-Werten}

Die HBM-Kommission hat die Voraussetzungen formuliert, die erfüllt sein müssen, um einen HBM-Wert auf Basis bereits vorliegender tolerabler Aufnahmemenge (TDI) abzuleiten [68]. In einem neueren Grundsatzpapier hat die Kommission darüber hinaus Kriterien für ein Verfahren formuliert, nach dem HBMWerte auch ohne vorliegende TDI-Werte abgeleitet werden können [69].

\subsection{Voraussetzung 1: Analytische Bestimmungsmethode}

Zur Analyse und Quantifizierung von NMP-Metaboliten im Urin liegt eine spezifische Methode vor [19], die für das „Human-Biomonitoring” der Allgemeinbevölkerung optimiert wurde [70]. Hierbei erfolgt im Anschluss an eine Festpha- senextraktion die gaschromatographische Trennung und der Nachweis mittels Tandem-Massenspektrometrie mit einer Quantifizierung über Isotopenverdünnung. Die Nachweisgrenze (LOD) für 5-HNMP und 2-HMSI liegt jeweils bei $1 \mu \mathrm{g} / \mathrm{l}$, die Bestimmungsgrenze (LOQ) jeweils bei 2,5 $\mu \mathrm{g} / \mathrm{l}$. Die Empfindlichkeit der Methode reicht aus, um Daten zur inneren Belastung der Allgemeinbevölkerung zu erheben.

\subsection{Voraussetzung 2: Kinetische Basisdaten}

Zur Toxikokinetik und zum Metabolismus von NMP bei Ratten und beim Menschen liegen valide Daten vor (siehe Kapitel 4.1).

Die Erfassung der inneren Belastung gegenüber NMP am Arbeitsplatz anhand der Ausscheidung von NMP-Metaboliten ist ein im Arbeitsschutz seit Langem etabliertes Verfahren [8, 71, 72]. Mit dem von Schindler et al. [19] beschriebenen Verfahren wurden bereits erste Befunde zur Ausscheidung von NMP-Metaboliten im Urin nicht beruflich belasteter Personen erhoben [17-19]. Weiterführende Daten zur Exposition der Allgemeinbevölkerung werden im Rahmen der derzeit laufenden Deutschen Umweltstudie zur Gesundheit von Kindern und Jugendlichen, GerES 2014 bis 2017 [73] erhoben.

\subsection{Voraussetzung 3: Vorliegen einer geeigneten Schlüsselstudie zur Toxizität}

Für die Ableitung von HBM-Werten auf Basis von Humanstudien ist die Datenlage unzureichend. Die vorliegenden Daten tierexperimenteller Studien zur Toxizität von NMP sowie die Tierversuchs- und Humandaten zur Toxikokinetik bilden jedoch eine ausreichende Bewertungsbasis zur Ableitung von HBM-Werten.

Kritische Effekte, die für die Ableitung von HBM-Werten als bewertungsrelevant angesehen werden, sind die in Tierversuchen beobachteten allgemein toxischen Effekte, insbesondere auf die Gewichtsentwicklung, sowie die in Studien zur Reproduktionstoxizität beobachteten embryo/fötoxischen und teratogenen Effekte bei gleichzeitig schwacher maternal 
toxischer Wirkung. Dabei traten in den entsprechenden Untersuchungen maternal toxische Wirkungen zumeist bereits bei Dosierungen auf, die noch keine reproduktionstoxischen oder/und teratogenen Wirkungen zeigten. Die genauere Betrachtung der reproduktionstoxischen und insbesondere der teratogenen Effekte weist aber auch darauf hin, dass diese nicht durch die maternale Toxizität hervorgerufen werden, sondern als davon unabhängige, eigenständige Wirkungen des NMP anzusehen sind $[15,16]$.

Gemäß Grundsatzpapier sollte als Schlüsselstudie bevorzugt eine Studie ausgewählt werden, die den Expositionsweg verwendet, auf dem die wirkungsbestimmende innere Exposition des Menschen erfolgt. Für NMP ist davon auszugehen, dass in erster Linie die inhalative, daneben die dermale Exposition bestimmend sein dürfte.

In einer Inhalationsstudie mit chronischer Exposition wurde eine NOAEC von $40 \mathrm{mg} / \mathrm{m}^{3}$ (umgerechnet auf kontinuierliche Exposition: $7,1 \mathrm{mg} / \mathrm{m}^{3}$ ) ermittelt [40]. Dabei erfolgte eine Ganzkörperexposition der Tiere. NMP wird dermal (auch in Dampfform, siehe Kapitel. 4.1) und oral sehr gut resorbiert, sodass über diese Pfade eine zusätzliche innere Belastung mit NMP zu vermuten ist. Die tatsächliche innere Exposition der Tiere dürfte somit höher gewesen sein, kann jedoch nicht näher quantifiziert werden. Außerdem war der Abstand zur nächst höheren Konzentration und damit zur LOAEC $\left(400 \mathrm{mg} / \mathrm{m}^{3}\right.$, kontinuierlich $71 \mathrm{mg} / \mathrm{m}^{3}$ ) groß, sodass der NOAEC dieser Studie als sehr konservativ einzuschätzen ist. Aus den genannten Gründen werden diese Befunde nicht zur Ableitung von HBM-Werten herangezogen.

In einer subchronischen Inhalationsstudie an Ratten mit Exposition gegenüber NMP-Dampf-Aerosolgemisch [41] erfolgte eine „Head-only-Exposition“, wodurch eine dermale und orale (durch Putzen des Fells erfolgende) Aufnahme weitestgehend vermieden wird. Die NOAEC lag in dieser Untersuchung bei $500 \mathrm{mg} / \mathrm{m}^{3}$ (umgerechnet auf kontinuierliche Exposition: $90 \mathrm{mg} /$ $\mathrm{m}^{3}$ ), die LOAEC bei $1000 \mathrm{mg} / \mathrm{m}^{3}$ (kontinuierlich: $180 \mathrm{mg} / \mathrm{m}^{3}$ ). Die NOAEC entspricht unter Standardannahmen [74] zu Körpergewicht und Atemrate von männ- lichen $\left(0,5 \mathrm{~kg}, 0,377 \mathrm{~m}^{3} / \mathrm{d}\right)$ bzw. weiblichen Ratten $\left(0,35 \mathrm{~kg}, 0,223 \mathrm{~m}^{3} / \mathrm{d}\right)$ und bei Annahme vollständiger Resorption einer zugeführten Dosis von etwa $90-100 \mathrm{mg} /(\mathrm{kg}$ KG $\times$ d), die LOAEC etwa 180-200 mg/(kg $\mathrm{KG} \times \mathrm{d})$.

In einer Inhalationsstudie zur Entwicklungstoxizität [57] lag die NOAEC (für maternal toxische Effekte) bei $124 \mathrm{mg} / \mathrm{m}^{3}$ (kontinuierlich $31 \mathrm{mg} / \mathrm{m}^{3}$ ), die LOAEC (die hinsichtlich entwicklungstoxischer Effekte eine NOAEC darstellt) bei $247 \mathrm{mg} / \mathrm{m}^{3}$ (kontinuierlich $62 \mathrm{mg} / \mathrm{m}^{3}$ ). Mit den genannten Standardannahmen [74] entspricht diese NOAEC/LOAEC Körperdosen von ca. 30 bzw. 60 mg/(kg $\mathrm{KG} \times \mathrm{d}$ ). Allerdings erfolgte in dieser Studie eine Ganzkörperexposition der Tiere, sodass zur inhalativen Exposition noch eine dermale und orale in nicht quantifizierbarer Höhe hinzukommt.

Eine prinzipiell denkbare Ableitung auf Basis tierexperimenteller Studien mit dermaler Verabreichung wird wegen der für diesen Pfad eingeschränkten Datenlage und Schwierigkeiten bei der Extrapolation, auch hinsichtlich Unterschieden in der dermalen Resorption bei Nagern und beim Menschen als zu unsicher angesehen.

Wegen der Unsicherheiten bei der Umrechnung der NMP-Konzentration in Luft bei inhalativer Exposition auf zugeführte Körperdosen und der Unsicherheiten über die Höhe einer zusätzlichen dermalen und oralen Aufnahme werden zur Ableitung von HBM-Werten Befunde von Studien mit oraler Exposition herangezogen und Befunde aus Inhalationsstudien als stützend betrachtet.

Als kritische Studie zur Ableitung der HBM-Werte wird zum einen die von Saillenfait et al. [53] durchgeführte Studie zur Entwicklungstoxizität mit oraler Verabreichung von NMP an trächtige Ratten herangezogen, zum anderen die Studie von Sitarek et al. [50] zur Fertilität weiblicher Ratten und der Entwicklung ihrer Nachkommen.

In der Studie von Saillenfait et al. [53] lag der NOAEL für maternal und entwicklungstoxische Effekte bei $125 \mathrm{mg} /$ $(\mathrm{kg} \mathrm{KG} \times \mathrm{d})$ und damit nur knapp unterhalb der Dosis von $150 \mathrm{mg} /(\mathrm{kg} \mathrm{KG} \times \mathrm{d})$, bei der in der Untersuchung von Sitarek et al. [50] bereits maternal und entwick- lungstoxische Effekte auftraten. Ein NOAEL wurde in der Studie von Sitarek et al. [50] nicht ermittelt.

Es erscheint daher gerechtfertigt, auf den NOAEL der Untersuchung von Saillenfait et al. [50] einen zusätzlichen Extrapolationsfaktor von 3 anzuwenden, um den Abstand zwischen dem LOAEL aus der Studie von Sitarek et al. [50] und dem POD zur Ableitung des HBM-I-Werts zu vergrößern. Die Anwendung eines solchen Faktors ist prinzipiell nach dem Grundsatzpapier zur Ableitung von HBM-Werten [69] möglich. Der resultierende POD von $42 \mathrm{mg} /(\mathrm{kg} \mathrm{KG} \times \mathrm{d})$ liegt um einen Faktor von etwa 3,5 unter dem LOAEL der genannten Studie von Sitarek et al. [50] und damit in einem Abstand zum LOAEL, wie er auch bei der Extrapolation eines NOAEL aus einem LOAEL durch einen ,assessment factor" von 3 erreicht wird.

Der POD von $42 \mathrm{mg} /(\mathrm{kg} \mathrm{KG} \times \mathrm{d})$ wird weiterhin gestützt durch die oben genannten Betrachtungen zur Pfad-zuPfad-Extrapolation der Ergebnisse einer Inhalationsstudie zur Entwicklungstoxizität [57], die eine NOAEC von ca. $30 \mathrm{mg}$ / $(\mathrm{kg} \mathrm{KG} \times \mathrm{d})$ ergaben, wobei aber die infolge Ganzkörperexposition erfolgte zusätzliche dermale und orale Aufnahme nicht berücksichtigt ist.

Somit ergibt sich:

\subsection{Ableitung des HBM-I-Wertes}

POD (NOAEL): $125 \mathrm{mg} /(\mathrm{kg} \mathrm{KG} \times d)$

Extrapolationsfaktoren:

Zeitextrapolation: Entfällt

Interspeziesextrapolation (Allometrie, Toxikodyna-

mik): 4 × 2,5 = 10

Intraspeziesvariabilität:

Dosis-Wirkungs-Beziehung,

Abstand NOAEL(Saillenfait-Studie)

zu LOAEL(Sitarek-Studie) : 3

Gesamtfaktor

- Tolerable externe Körperdosis, Mensch: $125 \mathrm{mg} /(\mathrm{kg} \mathrm{KG} \times \mathrm{d}): 300=0,42 \mathrm{mg} /(\mathrm{kg}$ $K G \times d)$

Bezug auf Metabolitenausscheidung im Urin: Die Molmasse von NMP beträgt 99,1 g/mol, die der beiden Metaboliten 5-HNMP und 2HMSI 115,1 bzw. 129,1 g/ 
mol, gemittelt 122,1. Das Verhältnis der Molmassen Metaboliten (gemittelt)/: NMP beträgt somit 122,1:99,1=1,23. Der Konversionsfaktor fue beträgt 0,65 (siehe Kap. 4.1.2).

Es gilt: tolerable $\Sigma$ (5-HNMP + 2-HMSI) im Urin = tolerable externe Körperdosis $\times$ (Mittelwert Molmasse Metaboliten/Molmasse NMP) $\times$ fue

$$
\begin{aligned}
& \text { Tolerable } \sum(5-H N M P+2-H M S I) ~ i m \\
& \text { Urin }=0,42 \times 1,23 \times 0,65=0,34 \mathrm{mg} /(\mathrm{kg} \\
& K G \times d)
\end{aligned}
$$

Wird die berechnete Ausscheidung vereinfachend auf eine körpergewichtsproportionale Urinmenge von 0,03 L/(kg KG $\times$ d) bei Kindern bzw. 0,02 L/( $\mathrm{kg} \mathrm{KG} \times \mathrm{d})$ bei Erwachsenen bezogen, so ergeben sich als HBM-I-Werte für die Summe der beiden Metaboliten 5-HNMP + 2-HMSI:

- HBM-I-Wert, Urin, Kinder:

11,2 mg/L (gerundet: $10 \mathrm{mg} / \mathrm{L}$ )

- HBM-I-Wert, Urin, Erwachsene:

16,8 mg/L (gerundet: $15 \mathrm{mg} / \mathrm{L}$ )

\subsection{Ableitung des HBM-II-Wertes}

Für die Ableitung des HBM-II-Wertes wird auf den NOAEL von $125 \mathrm{mg} /(\mathrm{kg} \mathrm{KG} \times$ d) aus der Studie von Saillenfait et al. [53] rekurriert. Dieser Wert liegt knapp unterhalb des LOAEL von $150 \mathrm{mg} /(\mathrm{kg} \mathrm{KG} \times$ d), der in der Fertilitätsstudie von Sitarek et al. [50] bereits erste adverse Effekte erkennen ließ, ein NOAEL wurde in der letztgenannten Studie nicht ermittelt.

Es ergibt sich:

- HBM-II-Wert, Urin, Kinder, gerundet: $30 \mathrm{mg} / \mathrm{L}$

- HBM-II-Wert, Urin, Erwachsene, gerundet: $50 \mathrm{mg} / \mathrm{L}$

\section{Summenbewertung NMP und NEP}

Die genannten HBM-I- und HBM-IIWerte gelten für eine Einzelstoffbetrachtung. In der Praxis ist neben einer Belastung mit NMP stets auch eine zeitgleiche Belastung mit NEP in Betracht zu ziehen. Da beide Stoffe ein sehr ähnliches Wirkungsprofil haben, insbesondere hinsichtlich entwicklungstoxischer und teratogener Effekte, ist die Mischexposition gegenüber beiden Stoffen bei der Bewertung von Befunden zu berücksichti- gen. Für den Gesamt HBM-I bzw. HBMII muss jeweils folgende Gleichung erfüllt sein:

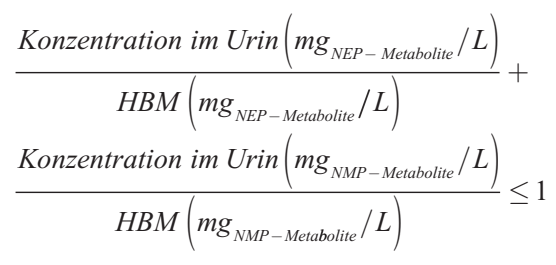

\section{Korrespondenzadresse}

Bekanntmachung des Umweltbundesamtes

Danksagung. Die HBM-Kommission dankt Dr. Jens-Uwe Voss (Müllheim) für die Erstellung des Manuskriptes, Petra Apel (Berlin), Dr. Holger M. Koch (Bochum) und Prof. Dr. Jürgen Angerer (Bochum) für die ergänzenden Beitrage sowie Angela Lehmann (Berlin) für die Unterstützung bei der redaktionellen Bearbeitung.

\section{Einhaltung ethischer Richtlinien}

Interessenkonflikt. Es bestand kein Interessenkonflikt bei der Ableitung o.g. HBM-Werte.

\section{Literatur}

1. Boenisch U, Bohme A, Kohajda T, Mögel I, Schütze N, von Bergen M, Simon JC, Lehmann I, Polte T (2012) Volatile organic compounds enhance allergic airway inflammation in an experimental mouse model. PLoS One 7(7):e39817, 1-14. (http://www.plosone.org/article/info\%3Adoi\%2F10.1371\%2Fjournal.pone.0039817)

2. Jouyban A, Fakhree MA, Shayanfar A (2010) Review of pharmaceutical applications of N-methyl2-pyrrolidone. J Pharm Pharm Sci 13(4):524-535. (https://ejournals.library.ualberta.ca/index.php/ JPPS/article/download/8257/7452)

3. Scientific Committee on Consumer Safety (SCCS) (2011) Opinion on N-Methyl-2-pyrrolidone. SCCS/1413/11,1-40 (Hrsg). European Commission Health \& Consumers, Directorate D: Health Systems and Products, Unit D3 - Risk Assessment, European Union, Brussels, Belgium. http://ec.europa.eu/health/scientific_committees/consumer_ safety/docs/sccs_o_050.pdf

4. Ad-hoc-AG des Umweltbundesamtes (2014) Richtwerte für 1-Methyl-2-pyrrolidon in der Innenraumluft. Bundesgesundheitsblatt Gesundheitsforschung Gesundheitsschutz 57(10):1232-1241. (http://link.springer.com/article/10.1007/s00103014-2041-1)

5. Deutsche Forschungsgemeinschaft (DFG) (1994) N-Methyl-2-pyrrolidon (Dampf). Gesundheitsschädliche Arbeitsstoffe. Toxikologisch-arbeitsmedizinische Begründungen von MAK-Werten, 20. Lieferung. Wiley-VCH, Weinheim, S 1-2

6. Deutsche Forschungsgemeinschaft (DFG) (2002) N-Methyl-2-pyrrolidon (Dampf). Gesundheitsschädliche Arbeitsstoffe. Toxikologisch-arbeitsmedizinische Begründungen von MAK-Werten, 34. Lieferung. Wiley-VCH, Weinheim, S 1-2
7. Deutsche Forschungsgemeinschaft (DFG) (2006) N-Methyl-2-pyrrolidon (Dampf). Gesundheitsschädliche Arbeitsstoffe. Toxikologisch-arbeitsmedizinische Begründungen von MAK-Werten, 41. Lieferung. Wiley-VCH, Weinheim, S 1-25

8. Deutsche Forschungsgemeinschaft (DFG) (2008) N-Methyl-2-pyrrolidon. In: Greim, H. (ed.): Grenzwerte in biologischem Material, 15. Lieferung. Wiley-VCH, Weinheim, S 1-13

9. Deutsche Forschungsgemeinschaft (DFG) (2012) N-Methyl-2-pyrrolidon (Dampf). Gesundheitsschädliche Arbeitsstoffe. Toxikologisch-arbeitsmedizinische Begründungen von MAK-Werten, 52. Lieferung. Wiley-VCH, Weinheim, S 1-6

10. Organisation for Economic Co-operation and Development (OECD) (2007) 1-methyl pyrrolidone. SIDS Initial Assessment Report for SIAM 24, 17-20 April, Paris, France. http://www.oecd.org/chemicalsafety/risk-assessment/publishedassessments. htm

11. RAC (2011) Annex 1. Background document to the Opinion proposing harmonised classification and labelling at Community level of N-ethyl-2-pyrrolidone (NEP). ECHA/RAC/CLH-0-0000002192-83-01/ A1. Committee for Risk Assessment (RAC), European Chemicals Agency (ECHA), http://www.echa. europa.eu/documents/10162/3a82ebe5-301b4b5b-94b9-5d3d09a65dd0

12. SCOEL (2007) Recommendation from the Scientific Committee on Occupational Exposure Limits for N-Methyl-2-Pyrrolidone. SCOEL/SUM/119. European Commission, Employment, Social Affairs \& Inclusion, Health and safety at work. The Scientific Committee on Occupational Exposure Limits (SCOEL), http://ec.europa.eu/social/BlobServlet?do$\mathrm{cld}=3867$ \&langld=en

13. World Health Organization (WHO) (2001) N-Methyl-2-pyrrolidone. Concise International Chemical Assessment Document 35. WHO Press, Geneva. (http://www.who.int/ipcs/publications/cicad/en/ cicad35.pdf)

14. Europäische Chemikalienagentur (ECHA) (2013) 1-methyl-2-pyrrolidone. (ECHA), Annankatu 18, P.O. Box 400, FI-00121 Helsinki, Finnland.

15. National Institute for Public Health and the Environment (RIVM) (2013) Annex XV Restriction Report. Proposal for a Restriction. Substance Name: $\mathrm{N}$-methyl-2-pyrrolidone (NMP). Version number 2.0. Bureau REACH (Ed.). The Netherlands. http:// echa.europa.eu/documents/10162/ee4c88a9d26f-4872-98fd-fb41646cc9e1

16. National Institute for Public Health and the Environment (RIVM) (2013) CLH report. Proposal for Harmonised Classification and Labelling Based on Regulation (EC) No 1272/2008 (CLP Regulation), Annex VI, Part 2. Substance Name: 1-methyl-2-pyrrolidone. Version number 2. Bureau REACH (Ed.). The Netherlands. http://echa.europa.eu/ documents/10162/9a6bb3e4-6265-41d2-b96300e37f95a20e

17. Koslitz S, Meier S, Schindler BK, Koch HM, Weiss T, Brüning T, Käfferlein HU (2011) Erste Hinweise auf eine Hintergrundbelastung der Allgemeinbevölkerung mit N-Methyl-2-pyrrolidon und N-Ethyl-2pyrrolidon. Dokumentation der 51. Jahrestagung der DGAUM. 51. Triebig G (Hrsg). Aachen. http:// www.ipa.ruhr-uni-bochum.de/image/poster/330. pdf 
18. Weiß T, Meier S, Koslitz S, Schindler B, Koch HM, Käfferlein HU, Brüning T (2012) Innere Belastung mit N-Methyl-2-pyrrolidon (NMP) und N-Ethyl-2pyrrolidon (NEP) bei Lackierern aus der Automobilindustrie und beruflich nicht exponierten Kontrollen. Konferenz: 52. Wissenschaftiche Jahrestagung der Gesellschaft für Arbeitsmedizin und Umweltmedizin 14.-17. März 2012, Göttingen, Vortrag, Abstractband V 8,56-57. http://www.dgaum. de/dgaum-jahrestagung/archiv-jahrestagungen/ jahrestagung-2012/\#c199

19. Schindler BK, Koslitz S, Meier S, Belov VN, Koch HM, Weiss T, Brüning T, Käfferlein HU (2012) Quantification of four major metabolites of embryotoxic N-methyl- and N-ethyl-2-pyrrolidone in human urine by cooled-injection gas chromatography and isotope dilution mass spectrometry. Anal Chem 84(8):3787-3794. (http://pubs.acs.org/doi/ abs/10.1021/ac300439w)

20. Bundesanstalt für Arbeitsschutz und Arbeitsmedizin (BAuA) Ausschuss für Gefahrstoffe (AGS) (2011) Begründung für die Festlegung der Schwangerschaftskategorie für N-Methyl-2-pyrrolidon. http:// www.baua.de/de/Themen-von-A-Z/Gefahrstoffe/ TRGS/pdf/900/900-n-methyl-2-pyrrolidon.pdf? blob $=$ publicationFile $\& \mathrm{v}=1$

21. Wells DA, Digenis GA (1988) Disposition and metabolism of double-labeled [3H and 14C] N-methyl-2-pyrrolidinone in the rat. Drug Metab Dispos 16(2):243-249. (http://dmd.aspetjournals.org/content/16/2/243.abstract)

22. Sitarek K, Kilanowicz A (2006) Tissue distribution and excretion of $\mathrm{N}$-methyl-2-pyrrolidone in male and female rats. Int J Occup Med Environ Health 19(2):142-148. (http://www.ncbi.nlm.nih.gov/ pubmed/17128812)

23. Åkesson B, Jönsson BAG (1997) Major metabolic pathway for N-methyl-2-pyrrolidone in humans. Drug Metab Dispos 25(2):267-269. (http://dmd. aspetjournals.org/content/25/2/267.long)

24. Carnerup MA, Spanne M, Jönsson BAG (2006) Levels of N-methyl-2-pyrrolidone (NMP) and its metabolites in plasma and urine from volunteers after experimental exposure to NMP in dry and humid air. Toxicol Lett 162(2-3):139-145. (http:// www.sciencedirect.com/science/article/pii/ S0378427405002985)

25. Åkesson B, Jönsson BAG (2000) Biological monitoring of N-methyl-2-pyrrolidone using 5-hydro$\mathrm{xy}-\mathrm{N}$-methyl-2-pyrrolidone in plasma and urine as the biomarker. Scand J Work Environ Health 26(3):213-218. (http://www.sjweh.fi/show_abstract.php?abstract_id=534)

26. Bader M, Wrbitzky R, Blaszkewicz M, Schäper M, van Thriel C (2008) Human volunteer study on the inhalational and dermal absorption of N-methyl2-pyrrolidone (NMP) from the vapour phase. Arch Toxicol 82(1):13-20. (http://link.springer.com/article/10.1007\%2Fs00204-007-0230-5)

27. Åkesson B, Paulsson K (1997) Experimental exposure of male volunteers to N-methyl-2-pyrrolidone (NMP): acute effects and pharmacokinetics of NMP in plasma and urine. Occup Environ Med 54(4):236-240. (http://oem.bmj.com/content/54/4/236.full.pdf+html)

28. Jönsson BAG, Åkesson B (2003) Human experimental exposure to $\mathrm{N}$-methyl-2-pyrrolidone (NMP): toxicokinetics of NMP, 5-hydroxy- N-methyl-2-pyrrolidone, $\mathrm{N}$-methylsuccinimide and 2-hydroxy- $\mathrm{N}$-methylsuccinimide (2-HMSI), and biological monitoring using 2-HMSI as a biomarker. Int Arch Occup Environ Health 76(4):267-274. (http:// link.springer.com/article/10.1007/s00420-0030438-5)
29. Bader M, Wrbitzky R, Blaszkewicz M, van Thriel C (2007) Human experimental exposure study on the uptake and urinary elimination of N-methyl-2-pyrrolidone (NMP) during simulated workplace conditions. Arch Toxicol 81(5):335-346. (http://link.springer.com/article/ 10.1007\%2Fs00204-006-0161-6)

30. Poet TS, Kirman CR, Bader M, van Thriel C, Gargas ML, Hinderliter PM (2010) Quantitative risk analysis for N-methyl pyrrolidone using physiologically based pharmacokinetic and benchmark dose modeling. Toxicol Sci 113(2):468-482. (http://toxsci. oxfordjournals.org/content/113/2/468.long)

31. Kleinbeck S, Juran S, Schäper M, van Thriel C (2006) Veränderungen der Blinzelfrequenz durch akute Expositionen gegenüber N-Methyl-2-Pyrrolidon. Arbeitsmed Sozialmed Umweltmed 41(3):105106. (http://www.asu-arbeitsmedizin.com/Gentner.dll/asu-2006-03-093-192_MjA0NTQ5.PDF?UID $=8 B E 8$ AAEA0950CEC85A68A70AC0D75252DF95A 2F554B94CAA)

32. Kleinbeck S, Juran S, Schäper M et al (2006) Chemosensorische Empfindungen bei akuten Expositionen gegenüber N-Methyl-2-pyrrolidon (NMP) - Effekte von Expositionsspitzen und körperlichen Belastungen. Arbeitsmed Sozialmed Umweltmed 41(3):106-106

33. van Thriel C, Blaszkewicz M, Schaper M, Juran SA, Kleinbeck S, Kiesswetter E, Wrbitzky R, Stache J, Golka K, Bader M (2007) Chemosensory effects during acute exposure to N-methyl-2-pyrrolidone (NMP). Toxicol Lett 175(1-3):44-56. (http://www.sciencedirect.com/science/article/pii/ S0378427407009654)

34. Malek DE, Malley LA, Slone TW, Elliott GS, Kennedy GL, Mellert W, Deckardt K, Gembardt C, Hildebrand B, Murphy SR, Bower DB, Wright GA (1997) Repeated dose toxicity study (28 days) in rats and mice with N-methylpyrrolidone (NMP). Drug Chem Toxicol 20(1-2):63-77. (http://informahealthcare.com/ doi/abs/10.3109/01480549709011079?journalCo$\mathrm{de}=\mathrm{dct})$

35. BASF (1978) Unpublished results. Study XXV/436. BASF AG, Dept. of Toxicology. In: ECHA (2013) ANNEX XV RESTRICTION REPORT PROPOSAL FOR A RESTRICTION SUBSTANCE NAME(S): N-METHYLPYRROLIDONE (NMP) IUPAC NAME(S):1-METHYLPYRROLIDIN-2-ONE,EC NUMBER(S):212-828-1,CAS NUMBER(S): 872-50-4 http://echa.europa.eu/documents/10162/13641/nmp_annex_xv_report_ en.pdf

36. Malley LA, Kennedy GL, Elliott GS, Slone TW, Mellert W, Deckardt K, Gembardt C, Hildebrand B, Parod RJ, McCarthy TJ, Griffiths JC (1999) 90-day subchronic toxicity study in rats and mice fed N-methylpyrrolidone (NMP) including neurotoxicity evaluation in rats. Drug Chem Toxicol 22(3):455-480. (http://informahealthcare.com/doi/abs/10.3109/ 01480549909042526?journalCode=dct)

37. NMP Producers Group (1994) Repeated dose toxicity study with N-Methylpyrrolidone in B6C3F1 mice - Administration in the diet for 4 weeks (range-finding study). Department of Toxicology of BASF AG, Ludwigshafen, Germany, Project No. 40C0225/93030, unpublished report. In: ECHA (2013) ANNEX XV RESTRICTION REPORT PROPOSAL FOR A RESTRICTION SUBSTANCE NAME(S): NMETHYLPYRROLIDONE (NMP) IUPAC NAME(S):1METHYLPYRROLIDIN-2-ONE,EC NUMBER(S):212828-1,CAS NUMBER(S): 872-50-4 http://echa.europa.eu/documents/10162/13641/nmp_annex_xv_ report_en.pdf
38. Becci PJ, Gephart LA, Koschier FJ, Johnson WD, Burnette LW (1983) Subchronic feeding study in beagle dogs of $\mathrm{N}$-methylpyrrolidone. J Appl Toxicol 3(2):83-86. (http://onlinelibrary.wiley.com/ doi/10.1002/jat.2550030206/abstract)

39. BASF AG (1993) Department of Toxicology, unpublished results, project No. 40I0544/90067, 26 Oct 1993. In: ECHA (2013) ANNEX XV RESTRICTION REPORT PROPOSAL FOR A RESTRICTION SUBSTANCE NAME(S): N-METHYLPYRROLIDONE (NMP) IUPAC NAME(S):1-METHYLPYRROLIDIN-2-ONE,EC NUMBER(S):212-828-1,CAS NUMBER(S): 872-50-4 http://echa.europa.eu/documents/10162/13641/ nmp_annex_xv_report_en.pdf

40. Lee KP, Chromey NC, Culik R, Barnes JR, Schneider PW (1987) Toxicity of N-methyl-2-pyrrolidone (NMP): teratogenic, subchronic, and two-year inhalation studies. Fundam Appl Toxicol 9(2):222235. (http://toxsci.oxfordjournals.org/content/9/2/222.abstract)

41. BASF (1994) Study on the inhalation toxicity of Nmethylpyrrolidone as a liquid aerosol in rats. 90day test including an about 4-week post-exposure observation period. Project No. 4010544/90061, unpublished report. In: ECHA (2013) ANNEX XV RESTRICTION REPORT PROPOSAL FOR A RESTRICTION SUBSTANCE NAME(S): N-METHYLPYRROLIDONE (NMP) IUPAC NAME(S):1-METHYLPYRROLIDIN-2-ONE,EC NUMBER(S):212-828-1,CAS NUMBER(S): 872-50-4 http://echa.europa.eu/documents/10162/13641/nmp_annex_xv_report_ en.pdf

42. Industrial Biology Research and Testing Laboratories (IBRTL) (1963) Twenty day subacute dermal toxicity study in rabbits with GAF - Methyl-Pyrrolodone. ISP Inventory No. 1963-001-01. In: ECHA (2013) ANNEX XV RESTRICTION REPORT PROPOSAL FOR A RESTRICTION SUBSTANCE NAME(S): NMETHYLPYRROLIDONE (NMP) IUPAC NAME(S):1METHYLPYRROLIDIN-2-ONE,EC NUMBER(S):212828-1,CAS NUMBER(S): 872-50-4 http://echa.europa.eu/documents/10162/13641/nmp_annex_xv_ report_en.pdf

43. Malley LA, Kennedy GL, Elliott GS, Slone TW, Mellert W, Deckardt K, Kuttler K, Hildebrand B, Banton MI, Parod RJ, Griffiths JC (2001) Chronic toxicity and oncogenicity of N-methylpyrrolidone (NMP) in rats and mice by dietary administration. Drug Chem Toxicol 24(4):315-338. (http:// informahealthcare.com/doi/abs/10.1081/dct100106262?journalCode $=\mathrm{dct}$ )

44. Parod RJ, Kaufmann W, Deckardt K et al (2001) Liver tumours in mice - N-Methylpyrrolidone (NMP) acts via enhanced cell proliferation. The Toxicologist 60:286

45. Solomon GM, Morse EP, Garbo MJ, Milton DK (1996) Stillbirth after occupational exposure to $\mathrm{N}$ methyl-2-pyrrolidone. A case report and review of the literature. J Occup Environ Med 38(7):705713. (http://journals.lww.com/joem/pages/articleviewer.aspx?year $=1996 \&$ issue $=07000 \& a r t i c-$ le $=00014 \&$ type $=$ abstract $)$

46. Exxon Biomedical Sciences (Exxon) (1991) Multigeneration rat reproduction study with $\mathrm{N}$-methylpyrrolidone. Biomedical Science, Inc.; Study for GAFCorp. (USA) (Project No. 236535, 26 November 1991). In: World Health Organization (WHO) (2001) Concise International Chemical Assessment Document 35, N-METHYL-2-PYRROLIDONE http://www. who.int/ipcs/publications/cicad/en/cicad35.pdf. 
47. NMP Producers Group (1999) N-Methylpyrrolidone (NMP): Two generation reproduction toxicity study in Wistar rats - Administration in the diet. Department of Toxicology of BASF Aktiengesellschaft, Ludwigshafen, Germany, Project No. 70R0056/97008, unpublished report. In: ECHA (2013) ANNEX XV RESTRICTION REPORT PROPOSAL FOR A RESTRICTION SUBSTANCE NAME(S): NMETHYLPYRROLIDONE (NMP) IUPAC NAME(S):1METHYLPYRROLIDIN-2-ONE,EC NUMBER(S):212828-1,CAS NUMBER(S): 872-50-4 http://echa.europa.eu/documents/10162/13641/nmp_annex_xv_ report_en.pdf

48. NMP Producers group (1999c). Two-generation reproduction toxicity study with N-Methylpyrrolidone (NMP) in Sprague-Dawley rats - Administration in the diet. Huntingdon Life Science, East Millestone, NJ, USA, project No.: 97-4106, unpublished report. In: ECHA (2013) ANNEX XV RESTRICTION REPORT PROPOSAL FOR A RESTRICTION SUBSTANCE NAME(S): N-METHYLPYRROLIDONE (NMP) IUPAC NAME(S):1-METHYLPYRROLIDIN-2-ONE,EC NUMBER(S):212-828-1,CAS NUMBER(S): 872-50-4 http://echa.europa.eu/documents/10162/13641/ nmp_annex_xv_report_en.pdf

49. Sitarek K, Stetkiewicz J (2008) Assessment of reproductive toxicity and gonadotoxic potential of $\mathrm{N}$-methyl-2-pyrrolidone in male rats. Int J Occup Med Environ Health 21(1):73-80. (http://www. ncbi.nlm.nih.gov/pubmed/18468972)

50. Sitarek K, Stetkiewicz J, Wasowicz W (2012) Evaluation of reproductive disorders in female rats exposed to N-methyl-2-pyrrolidone. Birth Defects Res B Dev Reprod Toxicol 95(3):195-201. (http://onlinelibrary.wiley.com/doi/10.1002/bdrb.21001/pdf)

51. Fries $A S$, Hass $U$, Jakobsen $B M$, Jelnes JE, Lund $S P_{,}$ Simonsen L (1992) Toxic effects of N-methylpyrrolidone on foetal development, the central nervous system, testes and semen in rats. Report No. 790037, Arbejdsmiljödfondet, Kopenhagen In: Deutsche Forschungsgemeinschaft e. V. (DFG) The MAK Collection for Occupational Health and Safety, N-Methyl-2-pyrrolidone (vapour) H, Published Online: 31 JAN 2012 http://onlinelibrary.wiley.com/doi/10.1002/3527600418.mb87250dame0010/pdf

52. Solomon HM, Burgess BA, Kennedy GL Jr, Staples RE (1995) 1-Methyl-2-pyrrolidone (NMP): reproductive and developmental toxicity study by inhalation in the rat. Drug Chem Toxicol 18(4):271293. (http://informahealthcare.com/doi/ abs/10.3109/01480549509014324)

53. Saillenfait AM, Gallissot F, Langonné I, Sabaté JP (2002) Developmental toxicity of N-methyl-2-pyrrolidone administered orally to rats. Food Chem Toxicol 40(11):1705-1712. (http://www.sciencedirect.com/science/article/pii/S0278691502001151)

54. Saillenfait AM, Sabaté JP, Gallissot F (2007) Comparative developmental toxicities of the three major metabolites of $\mathrm{N}$-methyl-2-pyrrolidone after oral administration in rats. J Appl Toxicol 27(6):571581. (http://onlinelibrary.wiley.com/doi/10.1002/ jat.1238/abstract)

55. Exxon Biomedical Sciences (Exxon) (1992) Developmental toxicity study in rats with $\mathrm{N}$-methyl2-pyrrolidone. Project No. 136534, 05 Jun 1992 (sponsored by GAF Corp., Wayne, USA). In: ECHA (2013) ANNEX XV RESTRICTION REPORT PROPOSAL FOR A RESTRICTION SUBSTANCE NAME(S): NMETHYLPYRROLIDONE (NMP) IUPAC NAME(S):1METHYLPYRROLIDIN-2-ONE,EC NUMBER(S):212828-1,CAS NUMBER(S): 872-50-4 http://echa.europa.eu/documents/10162/13641/nmp_annex_xv_ report_en.pdf
56. International Research and Development Corp. (IRDC) (1991) Developmental toxicity study in New Zealand White rabbits. Testing laboratory: International Research and Development Corp. (IRDC) Report no.: 637- 002. Owner company: Atrix Laboratories, Inc. and GAF Chemicals Corporation. Report date: 1991-12-17. In: ECHA (2014) Committee for Risk Assessment RAC Annex 1 Background document to the Opinion proposing harmonised classification and labelling at Community level of 1-methyl-2-pyrrolidone (NMP) EC number: 212828-1CAS number: 872-50-4 CLH-0-000000406678-03/F http://echa.europa.eu/documents/10162/ fofd41ff-e1c1-4cc4-bc8b-7a4912cffdb2.

57. Saillenfait AM, Gallissot F, Morel G (2003) Developmental toxicity of $\mathrm{N}$-methyl-2-pyrrolidone in rats following inhalation exposure. Food Chem Toxicol 41(4):583-588. (http://www.sciencedirect.com/ science/article/pii/S0278691502003009)

58. BASF (1991) Range finding study for the maternal inhalation toxicity of $\mathrm{N}$-methylpyrrolidone in pregnant rabbits. Project no. 21R0544/90049. BASF AG, Dept. of Toxicology. Zitiert nach RIVM (2013a)

59. BASF (1993) Study of the prenatal toxicity of Nmethylpyrrolidone in rabbits after inhalation of vapour-aerosol mixtures. Project no. 41R0544/90100. BASF AG, Dept. of Toxicology. Zitiert nach RIVM (2013b)

60. Becci PJ, Knickerbocker MJ, Reagan EL, Parent RA, Burnette LW (1982) Teratogenicity study of N-methylpyrrolidone after dermal application to Sprague-Dawley rats. Fundam Appl Toxicol 2(2):73-76. (http://toxsci.oxfordjournals.org/content/2/2/73. abstract)

61. Food and Drug Research Laboratories (FDRL) (1979) Unpublished Report. Lab No. 161. Zitiert nach RIVM (2013b)

62. BASF. (1993) Unpublished results. Project no. 44R0544/90078. BASF AG, Dept. of Toxicology. Zitiert nach RIVM (2013a)

63. Amtsblatt der Europäischen Union (2008) L 353/1 Verordnung (EG) Nr. 1272/2008 des Europäischen Parlaments und des Rates über die Einstufung, Kennzeichnung und Verpackung von Stoffen und Gemischen, zur Änderung und Aufhebung der Richtlinien 67/548/EWG und 1999/45/EG und zur Änderung der Verordnung (EG) Nr. 1907/2006 http://eur-lex.europa.eu/LexUriServ/LexUriServ.do ?uri=OJ:L:2008:353:0001:1355:de:PDF

64. European Chemicals Agency (ECHA) (2012) Classification and Labelling Inventory: Harmonised Classification - Annex VI of Regulation (EC) No. 1272/2008 (CLP Regulation). European Chemicals Agency (ECHA). http://clp-inventory.echa.europa. eu/

65. European Chemicals Agency (ECHA) (2011) Inclusion of Substances of Very High Concern in the Candidate List (Decision of the European Chemicals Agency). DOC: ED/31/2011. Helsinki, Finland. http://echa.europa.eu/documents/10162/ b6b72fb4-fffo-43dd-be25-963428f8f726

66. European Chemicals Agency (ECHA) (2013) Candidate List of Substances of Very High Concern for Authorisation. 1-Methyl-2-pyrrolidone

67. Nielsen GD, Larsen ST, Olsen O, Løvik M, Poulsen LK, Glue C, Wolkoff P (2007) Do indoor chemicals promote development of airway allergy? Indoor Air 17(3):236-255. (http://onlinelibrary.wiley.com/ doi/10.1111/j.1600-0668.2006.00468.x/abstract)
68. Kommission Human-Biomonitoring des Umweltbundesamtes (2007) Ableitung von Human-Biomonitoring-(HBM-)Werten auf Basis tolerabler Aufnahmemengen - Teil II: Grundlagen und Ableitungsweg. Stellungnahme der Kommission Human-Biomonitoring des Umweltbundesamts. Bundesgesundheitsblatt Gesundheitsforschung Gesundheitsschutz 50(2):251-254. (http://link.springer.com/article/10.1007/s00103-007-0146-5)

69. Kommission Human-Biomonitoring des Umweltbundesamtes (2014) Grundsatzpapier zur Ableitung von HBM-Werten. Bundesgesundheitsblatt Gesundheitsforschung Gesundheitsschutz 57(1):138-147. (http://link.springer.com/article/ 10.1007\%2Fs00103-013-1867-2)

70. Bader M, Brodbeck T, Weiß T, Koch HM (2014) Human-Biomonitoring von N-Methyl-2-pyrrolidon (NMP) und N-Ethyl-2-pyrrolodon (NEP) im Urin mittels Gaschromatographie-Tandem-Massenspektrometrie. 54. Wissenschaftliche Jahrestagung der Deutschen Gesellschaft für Arbeitsmedizin und Umweltmedizin e. V.

71. Käfferlein HU, Meier S, Koslitz S, WeißT, Koch HM, Ronge T, Brüning T (2013) Exposition gegenüber entwicklungstoxischen N-Alkyl-2-pyrrolidonen. IPA J 01/2013:14-17. (http://www.ipa.ruhr-uni-bochum.de/pdf/IPA-Journal_1301_Pyrrolidone.pdf)

72. Meier S, Schindler BK, Koslitz S, Koch HM, Weiss T, Käfferlein HU, Brüning T. (2013) Biomonitoring of Exposure to N-Methyl-2-Pyrrolidone in Workers of the Automobile Industry. Ann Occup Hyg 57(6):766-773. (http://annhyg.oxfordjournals.org/ content/57/6/766.long)

73. http://www.umweltbundesamt.de/themen/gesundheit/belastung-des-menschen-ermitteln/umwelt-survey/5-umwelt-survey-von-2013-bis-2016

74. European Chemicals Agency (ECHA) (2012) Guidance on information requirements and chemical safety assessment. Chapter R.8: Characterisation of dose [concentration]-response for human health. European Chemicals Agency, Helsinki, Finland. http://echa.europa.eu/documents/10162/13632/ information_requirements_r8_en.pdf 